\title{
COMPONENT-BY-COMPONENT CONSTRUCTION OF GOOD INTERMEDIATE-RANK LATTICE RULES*
}

\author{
F. Y. $\mathrm{KUO}^{\dagger}$ AND S. $\mathrm{JOE}^{\ddagger}$
}

\begin{abstract}
It is known that the generating vector of a rank-1 lattice rule can be constructed component-by-component to achieve strong tractability error bounds in both weighted Korobov spaces and weighted Sobolev spaces. Since the weights for these spaces are nonincreasing, the first few variables are in a sense more important than the rest. We thus propose to copy the points of a rank-1 lattice rule a number of times in the first few dimensions to yield an intermediate-rank lattice rule. We show that the generating vector (and in weighted Sobolev spaces, the shift also) of an intermediate-rank lattice rule can also be constructed component-by-component to achieve strong tractability error bounds. In certain circumstances, these bounds are better than the corresponding bounds for rank-1 lattice rules.
\end{abstract}

Key words. quasi-Monte Carlo methods, intermediate-rank lattice rules, worst-case error, tractability

AMS subject classifications. 65D30, 65D32, 68Q25

DOI. $10.1137 /$ S0036142902407162

1. Introduction. The $d$-dimensional integral

$$
I_{d}(f)=\int_{[0,1]^{d}} f(\boldsymbol{x}) \mathrm{d} \boldsymbol{x}
$$

may be approximated using rank-1 lattice rules. These are equal-weight rules having quadrature points belonging to the set

$$
\left\{\left\{\frac{i \boldsymbol{z}}{n}\right\}: 0 \leq i \leq n-1\right\} .
$$

Here $\boldsymbol{z}$, known as the generating vector, is an integer vector having no factor in common with $n$, and the braces around a vector indicate that we take the fractional part of each component of the vector. It is shown in [14] that every lattice rule may be written as a multiple sum involving one or more generating vectors; the minimum number of generating vectors required to generate a lattice rule is known as the "rank" of the rule. Besides rank-1 lattice rules involving just one generating vector, there exist lattice rules having rank up to $d$. More information about lattice rules may be found in [11].

The construction of rank-1 lattice rules for integrands belonging to weighted Korobov and weighted Sobolev spaces has been studied in various papers. These weighted function spaces are tensor product reproducing kernel Hilbert spaces. Recall that a

*Received by the editors May 7, 2002; accepted for publication (in revised form) January 21, 2003; published electronically September 9, 2003.

http://www.siam.org/journals/sinum/41-4/40716.html

${ }^{\dagger}$ Department of Mathematics, The University of Waikato, Private Bag 3105, Hamilton, New Zealand. Current address: School of Mathematics, The University of New South Wales, Sydney, NSW 2052, Australia (fkuo@maths.unsw.edu.au).

${ }^{\ddagger}$ Department of Mathematics, The University of Waikato, Private Bag 3105, Hamilton, New Zealand (stephenj@math.waikato.ac.nz). 
quasi-Monte Carlo (QMC) rule

$$
Q_{n, d}(f)=\frac{1}{n} \sum_{i=0}^{n-1} f\left(\boldsymbol{x}_{i}\right)
$$

is an equal-weight quadrature rule with the quadrature points chosen in a deterministic way. The "worst-case error" of a QMC rule in some Hilbert space $H_{d}$ is defined to be

$$
e_{n, d}\left(Q_{n, d}, H_{d}\right):=\sup \left\{\left|Q_{n, d}(f)-I_{d}(f)\right|:\|f\|_{H_{d}} \leq 1, f \in H_{d}\right\},
$$

and the initial error is

$$
e_{0, d}\left(H_{d}\right):=\sup \left\{\left|I_{d}(f)\right|:\|f\|_{H_{d}} \leq 1, f \in H_{d}\right\} .
$$

Following the analysis by Sloan and Woźniakowski in [16], the integration problem is said to be "strongly QMC tractable" in the Hilbert space $H_{d}$ if the minimal number of function evaluations $n$ in a QMC rule (1.1) needed to reduce the initial error $e_{0, d}\left(H_{d}\right)$ by a factor of $\varepsilon>0$ is bounded by a polynomial in $\varepsilon^{-1}$ independently of $d$.

In [15], a component-by-component algorithm was developed for constructing rank-1 lattice rules in unweighted Korobov spaces. The algorithm was later extended to shifted rank-1 lattice rules (see [12]) in weighted Sobolev spaces, and the rules constructed achieve strong QMC tractability error bounds. Both these constructions assumed that $n$, the number of quadrature points, was a prime number. The construction was later generalized in [10] to rules with a composite number of points. Construction of rank-1 lattice rules in the randomized setting has been considered in [13]. Recently, it was shown in [9] that the constructions achieve the optimal rate of convergence in the corresponding function spaces.

Lattice rules constructed in this manner are "extensible" in terms of the dimension $d$; that is, if further dimensions are needed at a later stage, the additional components can be constructed with the existing components kept unchanged. However, if more points are required, then the rules need to be reconstructed from scratch. A recent work [4] showed the existence of good rank-1 lattice rules that are extensible both in terms of the number of points $n$ and the dimension $d$, but the proof is nonconstructive.

We are interested in "copying" rank-1 lattice rules. Since the weighted function spaces of interest have nonincreasing weights, the first few variables are in a sense more important than the rest. Therefore, it would seem intuitive to copy the points in the first few dimensions. Thus we may copy an $n$-point $d$-dimensional rank- 1 lattice rule $\ell$ times in each of the first $r$ dimensions, where $\ell \geq 1, \operatorname{gcd}(\ell, n)=1$, and $0 \leq r \leq d$. We then obtain the rule with $N=\ell^{r} n$ points given by

$$
Q_{n, d}(f)=\frac{1}{\ell^{r} n} \sum_{m_{r}=0}^{\ell-1} \ldots \sum_{m_{1}=0}^{\ell-1} \sum_{i=0}^{n-1} f\left(\left\{\frac{i \boldsymbol{z}}{n}+\frac{\left(m_{1}, \ldots, m_{r}, 0, \ldots, 0\right)}{\ell}\right\}\right) .
$$

We call the rule with these points "the $(\ell, r)$-copy of a rank-1 lattice rule with generating vector $\boldsymbol{z}$." When $r=0$ and/or $\ell=1$, we get just the original $n$-point rank-1 lattice rule. For $r \geq 1$, the resulting rule is a rank- $r$ lattice rule. These intermediaterank lattice rules have previously been considered in [7] and [8]. Typically, for reasons of tractability, we will take $r$ to be a fixed number, say, $r=1$, 2, or 3 . For the choice of $\ell$ it would seem reasonable on practical grounds and theoretical grounds (see Theorem 2.3 and Lemma 2.4) to take $\ell$ to be 2 in actual calculations. This value of $\ell=2$ has been used previously in [7] and [8]. 
Our plan is to construct intermediate-rank lattice rules in both weighted Korobov and weighted Sobolev spaces that achieve strong QMC tractability error bounds. In section 2, we consider intermediate-rank lattice rules in weighted Korobov spaces. We show that the intermediate-rank lattice rule we consider has the same worst-case error as a certain rank-1 lattice rule in a slightly different weighted Korobov space. We then show that there exist intermediate-rank lattice rules with error bounds which are better than the corresponding bounds for rank-1 lattice rules with approximately the same number of points. Moreover, we shall see that the generating vectors constructed component-by-component satisfy strong QMC tractability bounds and achieve the optimal rate of convergence in weighted Korobov spaces. In section 3, we give a brief discussion on the construction of shifted intermediate-rank lattice rules in weighted Sobolev spaces. The final section, section 4, contains numerical results.

Throughout the paper, we will assume that $n$ is a prime number to simplify the analysis. More general results for any positive integer $n$ can be obtained by emulating the more complicated analysis found in [10]. When $n$ is a prime number, $\boldsymbol{z}$ can be chosen from $\mathbb{Z}_{n}^{d}$, where $\mathbb{Z}_{n}:=\{1,2, \ldots, n-1\}$.

2. Intermediate-rank lattice rules in weighted Korobov spaces. We are interested in the weighted Korobov spaces of periodic functions considered in [10]. These spaces are parameterized by a real parameter $\alpha>1$ and two sequences of positive weights $\boldsymbol{\beta}=\left\{\beta_{j}\right\}$ and $\boldsymbol{\gamma}=\left\{\gamma_{j}\right\}$ satisfying

$$
\frac{\gamma_{1}}{\beta_{1}} \geq \frac{\gamma_{2}}{\beta_{2}} \geq \cdots
$$

The inner product in these spaces is given by

$$
\langle f, g\rangle_{d}=\sum_{\boldsymbol{h} \in \mathbb{Z}^{d}}\left(\hat{f}(\boldsymbol{h}) \overline{\hat{g}(\boldsymbol{h})} \prod_{j=1}^{d} r\left(\alpha, \beta_{j}, \gamma_{j}, h_{j}\right)\right),
$$

where

$$
r(\alpha, \beta, \gamma, h)= \begin{cases}\beta^{-1} & \text { if } h=0 \\ \gamma^{-1}|h|^{\alpha} & \text { otherwise }\end{cases}
$$

Here $\alpha$ is a smoothness parameter characterizing the rate of decay of the Fourier coefficients. Various variations of these spaces have previously been considered in works such as [5], [6], [15], and [16]. The worst-case error in these Korobov spaces for a $\mathrm{QMC}$ rule (1.1) is given by

$$
e_{n, d}^{2}\left(\boldsymbol{x}_{0}, \ldots, \boldsymbol{x}_{n-1}\right)=-\prod_{j=1}^{d} \beta_{j}+\frac{1}{n^{2}} \sum_{i=0}^{n-1} \sum_{k=0}^{n-1} \prod_{j=1}^{d}\left(\beta_{j}+\gamma_{j} \sum_{h=-\infty}^{\infty} \frac{e^{2 \pi \mathrm{i} h\left(x_{i, j}-x_{k, j}\right)}}{|h|^{\alpha}}\right),
$$

where the ' on the sum indicates that we omit the $h=0$ term. This expression may be written in terms of Bernoulli polynomials if $\alpha$ is chosen to be a positive even number. The initial error is

$$
e_{0, d}=\prod_{j=1}^{d} \beta_{j}^{\frac{1}{2}}
$$


Following the analysis of tractability in [16], it is possible to show that if the weights satisfy

$$
\sum_{j=1}^{\infty} \frac{\gamma_{j}}{\beta_{j}}<\infty,
$$

then an upper bound for the square worst-case error of the form

$$
\frac{b}{n} \prod_{j=1}^{d}\left(\beta_{j}+a \gamma_{j}\right),
$$

where $a, b>0$ are bounded independently of $d$, is enough to ensure strong QMC tractability, with the rate of convergence being $O\left(n^{-1 / 2}\right)$. Moreover, the optimal rate of convergence $O\left(n^{-\alpha / 2+\delta}\right)$, for any $\delta>0$, can be achieved if the weights satisfy a stronger condition,

$$
\sum_{j=1}^{\infty}\left(\frac{\gamma_{j}}{\beta_{j}}\right)^{\frac{1}{\alpha-2 \delta}}<\infty .
$$

It is worth mentioning that the condition (2.2) is also necessary for strong QMC tractability (see $[6]$ ).

We now consider the $(\ell, r)$-copy of a rank-1 lattice rule with generating vector $\boldsymbol{z}$, that is, a rule with points belonging to the set

$$
\left\{\left\{\frac{i \boldsymbol{z}}{n}+\frac{\left(m_{1}, \ldots, m_{r}, 0, \ldots, 0\right)}{\ell}\right\}: 0 \leq i \leq n-1,0 \leq m_{1}, \ldots, m_{r} \leq \ell-1\right\},
$$

where $\ell \geq 1, \operatorname{gcd}(\ell, n)=1$, and $0 \leq r \leq d$. An expression for $e_{n, d, \operatorname{copy}(\ell, r)}(\boldsymbol{z})$, the worst-case error for such a rule, is given in the next lemma. Note that though this intermediate-rank lattice rule has $N=\ell^{r} n$ points, the lemma shows that the worstcase error may be calculated by using a rule having just $n$ points. We will explore this further in the next subsection.

LEMMA 2.1. We have

$$
\begin{aligned}
e_{n, d, \operatorname{copy}(\ell, r)}^{2}(\boldsymbol{z})=-\prod_{j=1}^{d} \beta_{j}+\frac{1}{n} \sum_{k=0}^{n-1} & {\left[\prod_{j=1}^{r}\left(\beta_{j}+\frac{\gamma_{j}}{\ell^{\alpha}} \sum_{h=-\infty}^{\infty} \frac{e^{2 \pi \mathrm{i} h \ell k z_{j} / n}}{|h|^{\alpha}}\right)\right.} \\
& \left.\times \prod_{j=r+1}^{d}\left(\beta_{j}+\gamma_{j} \sum_{h=-\infty}^{\infty} \frac{e^{2 \pi \mathrm{i} h k z_{j} / n}}{|h|^{\alpha}}\right)\right] .
\end{aligned}
$$

Proof. We have from (2.1) that

$$
\begin{aligned}
& e_{n, d, \operatorname{copy}(\ell, r)}^{2}(\boldsymbol{z})=-\prod_{j=1}^{d} \beta_{j}+\frac{1}{\ell^{2 r} n^{2}} \sum_{q_{r}=0}^{\ell-1} \cdots \sum_{q_{1}=0}^{\ell-1} \sum_{m_{r}=0}^{\ell-1} \cdots \sum_{m_{1}=0}^{\ell-1} \sum_{i=0}^{n-1} \sum_{k=0}^{n-1} \\
& \prod_{j=1}^{r}\left(\beta_{j}+\gamma_{j} \sum_{h=-\infty}^{\infty} \frac{e^{2 \pi \mathrm{i} h\left(\left\{\frac{i z_{j}}{n}+\frac{q_{j}}{\ell}\right\}-\left\{\frac{k z_{j}}{n}+\frac{m_{j}}{\ell}\right\}\right)}}{|h|^{\alpha}}\right) \\
&\left.\times \prod_{j=r+1}^{d}\left(\beta_{j}+\gamma_{j} \sum_{h=-\infty}^{\infty} \frac{e^{2 \pi \mathrm{i} h\left(\left\{\frac{i z_{j}}{n}\right\}-\left\{\frac{k z_{j}}{n}\right\}\right)}}{|h|^{\alpha}}\right)\right] .
\end{aligned}
$$


The second term can be written as

$$
\begin{aligned}
\frac{1}{n^{2}} \sum_{i=0}^{n-1} \sum_{k=0}^{n-1}[ & \prod_{j=1}^{r}\left(\frac{1}{\ell^{2}} \sum_{q=0}^{\ell-1} \sum_{m=0}^{\ell-1}\left(\beta_{j}+\gamma_{j} \sum_{h=-\infty}^{\infty} \frac{e^{2 \pi \mathrm{i} h\left((i-k) z_{j} / n+(q-m) / \ell\right)}}{|h|^{\alpha}}\right)\right) \\
& \left.\times \prod_{j=r+1}^{d}\left(\beta_{j}+\gamma_{j} \sum_{h=-\infty}^{\infty} \frac{e^{2 \pi \mathrm{i} h(i-k) z_{j} / n}}{|h|^{\alpha}}\right)\right] .
\end{aligned}
$$

For $0 \leq q, m \leq \ell-1$, the values of $(q-m) \bmod \ell$ are just 0 to $\ell-1$ in some order, with each value occurring $\ell$ times. Thus we have

$$
\begin{aligned}
& \frac{1}{\ell^{2}} \sum_{q=0}^{\ell-1} \sum_{m=0}^{\ell-1}\left(\beta_{j}+\gamma_{j} \sum_{h=-\infty}^{\infty} \frac{e^{2 \pi \mathrm{i} h\left((i-k) z_{j} / n+(q-m) / \ell\right)}}{|h|^{\alpha}}\right) \\
= & \frac{1}{\ell} \sum_{m=0}^{\ell-1}\left(\beta_{j}+\gamma_{j} \sum_{h=-\infty}^{\infty} \frac{e^{2 \pi \mathrm{i} h\left((i-k) z_{j} / n+m / \ell\right)}}{|h|^{\alpha}}\right) .
\end{aligned}
$$

Now since

$$
\sum_{m=0}^{\ell-1} e^{2 \pi \mathrm{i} h m / \ell}= \begin{cases}\ell & \text { if } h \text { is a multiple of } \ell \\ 0 & \text { otherwise }\end{cases}
$$

we have

$$
\begin{aligned}
& \frac{1}{\ell} \sum_{m=0}^{\ell-1}\left(\beta_{j}+\gamma_{j} \sum_{h=-\infty}^{\infty} \frac{e^{2 \pi \mathrm{i} h\left((i-k) z_{j} / n+m / \ell\right)}}{|h|^{\alpha}}\right) \\
= & \beta_{j}+\frac{\gamma_{j}}{\ell} \sum_{h=-\infty}^{\infty}\left(\frac{e^{2 \pi \mathrm{i} h(i-k) z_{j} / n}}{|h|^{\alpha}} \sum_{m=0}^{\ell-1} e^{2 \pi \mathrm{i} h m / \ell}\right) \\
= & \beta_{j}+\frac{\gamma_{j}}{\ell} \sum_{m=-\infty}^{\infty}\left(\frac{e^{2 \pi \mathrm{i} m \ell(i-k) z_{j} / n}}{|m \ell|^{\alpha}} \cdot \ell\right) \\
= & \beta_{j}+\frac{\gamma_{j}}{\ell^{\alpha}} \sum_{h=-\infty}^{\infty} \frac{e^{2 \pi \mathrm{i} h \ell(i-k) z_{j} / n}}{|h|^{\alpha}} .
\end{aligned}
$$

Thus (2.4) can be simplified to

$$
\begin{aligned}
\frac{1}{n^{2}} \sum_{i=0}^{n-1} \sum_{k=0}^{n-1}[ & \prod_{j=1}^{r}\left(\beta_{j}+\frac{\gamma_{j}}{\ell^{\alpha}} \sum_{h=-\infty}^{\infty} \frac{e^{2 \pi \mathrm{i} h \ell(i-k) z_{j} / n}}{|h|^{\alpha}}\right) \\
& \left.\times \prod_{j=r+1}^{d}\left(\beta_{j}+\gamma_{j} \sum_{h=-\infty}^{\infty} \frac{e^{2 \pi \mathrm{i} h(i-k) z_{j} / n}}{|h|^{\alpha}}\right)\right],
\end{aligned}
$$

which can be simplified even further to

$$
\frac{1}{n} \sum_{k=0}^{n-1}\left[\prod_{j=1}^{r}\left(\beta_{j}+\frac{\gamma_{j}}{\ell^{\alpha}} \sum_{h=-\infty}^{\infty} \frac{e^{2 \pi \mathrm{i} h \ell k z_{j} / n}}{|h|^{\alpha}}\right) \prod_{j=r+1}^{d}\left(\beta_{j}+\gamma_{j} \sum_{h=-\infty}^{\infty} \frac{e^{2 \pi \mathrm{i} h k z_{j} / n}}{|h|^{\alpha}}\right)\right],
$$

since for $0 \leq i, k \leq n-1$, the values of $(i-k) \bmod n$ are just 0 to $n-1$ in some order, with each value occurring $n$ times. This completes the proof. 
2.1. Relationship with rank-1 lattice rules based on worst-case error. Let us define the sequence $\bar{\gamma}$ by

$$
\bar{\gamma}_{j}:= \begin{cases}\frac{\gamma_{j}}{\ell^{\alpha}} & \text { if } 1 \leq j \leq r \\ \gamma_{j} & \text { otherwise }\end{cases}
$$

and set $\bar{z}$ to be the $d$-dimensional vector with components given by

$$
\bar{z}_{j}:= \begin{cases}\ell z_{j} & \text { if } 1 \leq j \leq r \\ z_{j} & \text { otherwise }\end{cases}
$$

Then the expression in Lemma 2.1 may be rewritten in the form

$$
e_{n, d, \operatorname{copy}(\ell, r)}^{2}(\boldsymbol{z})=-\prod_{j=1}^{d} \beta_{j}+\frac{1}{n} \sum_{k=0}^{n-1} \prod_{j=1}^{d}\left(\beta_{j}+\bar{\gamma}_{j} \sum_{h=-\infty}^{\infty} \frac{e^{2 \pi \mathrm{i} h k \bar{z}_{j} / n}}{|h|^{\alpha}}\right)=e_{n, d}^{2}(\overline{\boldsymbol{z}}, \bar{\gamma}) ;
$$

that is, the worst-case error of an intermediate-rank lattice rule with generating vector $\boldsymbol{z}$ in the weighted Korobov space with weights $\boldsymbol{\beta}$ and $\boldsymbol{\gamma}$ is the same as the worst-case error of a rank-1 lattice rule with generating vector $\overline{\boldsymbol{z}}$ in the weighted Korobov space with weights $\boldsymbol{\beta}$ and $\overline{\boldsymbol{\gamma}}$.

Since $\ell \neq 0$ and $\operatorname{gcd}(\ell, n)=1$, for fixed $r$ there exist a unique $\overline{\boldsymbol{z}}$ for each $\boldsymbol{z}$ and vice versa. Because of this one-to-one correspondence between $\boldsymbol{z}$ and $\overline{\boldsymbol{z}}$, all the known results on rank-1 lattice rules in weighted Korobov spaces can be applied here, with generating vector $\overline{\boldsymbol{z}}$ and weights $\boldsymbol{\beta}$ and $\overline{\boldsymbol{\gamma}}$. Note that the effect of copying in the first

$r$ dimensions can be interpreted as a reduction of the first $r$ terms of $\gamma$ by a factor of $1 / \ell^{\alpha}$.

The following theorem is a slight generalization of Lemma 2 in [16]. (There $\boldsymbol{\beta}$ is assumed to be 1.)

TheOREM 2.2. Let $n$ be a prime number, and define $M_{n, d, \operatorname{copy}(\ell, r)}$ to be the mean given by

$$
M_{n, d, \operatorname{copy}(\ell, r)}:=\frac{1}{(n-1)^{d}} \sum_{\boldsymbol{z} \in \mathbb{Z}_{n}^{d}} e_{n, d, \operatorname{copy}(\ell, r)}^{2}(\boldsymbol{z}) .
$$

Then an expression for $M_{n, d, \operatorname{copy}(\ell, r)}$ is given by

$$
\begin{aligned}
& -\prod_{j=1}^{d} \beta_{j}+\frac{1}{n} \prod_{j=1}^{r}\left(\beta_{j}+\frac{2 \gamma_{j} \zeta(\alpha)}{\ell^{\alpha}}\right) \prod_{j=r+1}^{d}\left(\beta_{j}+2 \gamma_{j} \zeta(\alpha)\right) \\
& +\left(1-\frac{1}{n}\right) \prod_{j=1}^{r}\left(\beta_{j}-\frac{2 \gamma_{j} \zeta(\alpha)\left(1-n^{1-\alpha}\right)}{(n-1) \ell^{\alpha}}\right) \prod_{j=r+1}^{d}\left(\beta_{j}-\frac{2 \gamma_{j} \zeta(\alpha)\left(1-n^{1-\alpha}\right)}{n-1}\right),
\end{aligned}
$$

where $\zeta(\alpha)$ is the Riemann zeta function. Moreover, if $n$ satisfies $n \geq 1+\frac{\gamma_{1}}{\beta_{1}} \zeta(\alpha)$, then

$$
M_{n, d, \operatorname{copy}(\ell, r)} \leq \frac{1}{n} \prod_{j=1}^{r}\left(\beta_{j}+\frac{2 \gamma_{j} \zeta(\alpha)}{\ell^{\alpha}}\right) \prod_{j=r+1}^{d}\left(\beta_{j}+2 \gamma_{j} \zeta(\alpha)\right) .
$$


Clearly there must exist at least one vector $\boldsymbol{z}$ such that

$$
e_{n, d, \operatorname{copy}(\ell, r)}^{2}(\boldsymbol{z}) \leq M_{n, d, \operatorname{copy}(\ell, r)} \leq \frac{1}{n} \prod_{j=1}^{d}\left(\beta_{j}+2 \gamma_{j} \zeta(\alpha)\right) .
$$

Now let $N=\ell^{r} n$ denote the total number of quadrature points. It is obvious that this last bound is of the form (2.3) with $a=2 \zeta(\alpha), b=\ell^{r}$, and $n=N$. Since $\ell$ and $r$ are fixed, we conclude that there exist intermediate-rank lattice rules that achieve strong QMC tractability error bounds for weighted Korobov spaces.

2.2. Comparison with rank-1 lattice rules based on mean. It follows from Theorem 2.2 with $\ell=1$ and $n=N$ that for $N$ prime, the mean for rank-1 lattice rules is

$\widehat{M}_{N, d}=-\prod_{j=1}^{d} \beta_{j}+\frac{1}{N} \prod_{j=1}^{d}\left(\beta_{j}+2 \gamma_{j} \zeta(\alpha)\right)+\left(1-\frac{1}{N}\right) \prod_{j=1}^{d}\left(\beta_{j}-\frac{2 \gamma_{j} \zeta(\alpha)\left(1-N^{1-\alpha}\right)}{N-1}\right)$.

Suppose we replace $N$ by $N=\ell^{r} n$ in this last expression. This is not valid because $N$ is not prime, but calculations using the correct (but more complicated) expression for the mean found in [10] indicate that this yields an underestimate of the true mean.

Now let

$$
R_{n, d, \ell, r}:=\frac{M_{n, d, \operatorname{copy}(\ell, r)}}{\widehat{M}_{N, d}} .
$$

As an indication of whether these intermediate-rank lattice rules are better than rank-1 lattice rules having approximately the same number of points, we would like a result which shows that $R_{n, d, \ell, r}<1$. A preliminary result of this type is given in the following theorem.

THEOREM 2.3. Suppose that $n$ is a prime number satisfying $n \geq 1+\frac{2 \gamma_{1}}{\beta_{1}} \zeta(\alpha)$. If

$$
\rho_{\ell, r}:=\prod_{j=1}^{r} \frac{\ell \beta_{j}+\frac{2 \gamma_{j} \zeta(\alpha)}{\ell^{\alpha-1}}}{\beta_{j}+2 \gamma_{j} \zeta(\alpha)}<1
$$

and

$$
\begin{aligned}
& \ell^{r}(n-1) \prod_{j=1}^{r}\left(\beta_{j}-\frac{2 \gamma_{j} \zeta(\alpha)\left(1-n^{1-\alpha}\right)}{(n-1) \ell^{\alpha}}\right) \prod_{j=r+1}^{d}\left(\beta_{j}-\frac{2 \gamma_{j} \zeta(\alpha)\left(1-n^{1-\alpha}\right)}{n-1}\right) \\
< & \left(\ell^{r} n-1\right) \prod_{j=1}^{d}\left(\beta_{j}-\frac{2 \gamma_{j} \zeta(\alpha)\left(1-\left(\ell^{r} n\right)^{1-\alpha}\right)}{\ell^{r} n-1}\right),
\end{aligned}
$$

then

$$
R_{n, d, \ell, r}<\rho_{\ell, r} .
$$

Proof. By multiplying both $M_{n, d, \operatorname{copy}(\ell, r)}$ and $\widehat{M}_{N, d}$ by $N=\ell^{r} n$, we can write

$$
R_{n, d, \ell, r}=\frac{t_{1}+t_{2}-c}{b_{1}+b_{2}-c} \quad \text { and } \quad \rho_{\ell, r}=\frac{t_{1}}{b_{1}}
$$


where

$$
\begin{aligned}
t_{1} & =\prod_{j=1}^{r}\left(\ell \beta_{j}+\frac{2 \gamma_{j} \zeta(\alpha)}{\ell^{\alpha-1}}\right) \prod_{j=r+1}^{d}\left(\beta_{j}+2 \gamma_{j} \zeta(\alpha)\right), \\
t_{2} & =\ell^{r}(n-1) \prod_{j=1}^{r}\left(\beta_{j}-\frac{2 \gamma_{j} \zeta(\alpha)\left(1-n^{1-\alpha}\right)}{(n-1) \ell^{\alpha}}\right) \prod_{j=r+1}^{d}\left(\beta_{j}-\frac{2 \gamma_{j} \zeta(\alpha)\left(1-n^{1-\alpha}\right)}{n-1}\right), \\
b_{1} & =\prod_{j=1}^{d}\left(\beta_{j}+2 \gamma_{j} \zeta(\alpha)\right), \\
b_{2} & =\left(\ell^{r} n-1\right) \prod_{j=1}^{d}\left(\beta_{j}-\frac{2 \gamma_{j} \zeta(\alpha)\left(1-\left(\ell^{r} n\right)^{1-\alpha}\right)}{\ell^{r} n-1}\right), \\
c & =\ell^{r} n \prod_{j=1}^{d} \beta_{j} .
\end{aligned}
$$

It is not hard to prove that

$$
\frac{t_{1}+t_{2}-c}{b_{1}+b_{2}-c}<\frac{t_{1}}{b_{1}}
$$

is true if $b_{1}, b_{2}, t_{1}, t_{2}$, and $c$ are positive quantities satisfying

$$
t_{1}<b_{1}, \quad b_{1}+b_{2}>c, \quad \text { and } t_{2}<b_{2}<c .
$$

Thus the result is proved if we can prove that all these conditions hold.

It may not be obvious that $b_{2}$ and $t_{2}$ are positive quantities, but one can see that this is the case when $\beta_{j}-2 \gamma_{j} \zeta(\alpha) /(n-1)>0$ for $j=1,2 \ldots, d$, which is equivalent to the requirement on $n$ given in the statement of the theorem. The requirement that $t_{1}<b_{1}$ comes from the assumption that $\rho_{\ell, r}<1$, while the requirement that $t_{2}<b_{2}$ comes from the assumption given in (2.7). Also, it is clear that $b_{2}<c$.

Let

$$
\hat{b}_{2}=\left(\ell^{r} n-1\right) \prod_{j=1}^{d}\left(\beta_{j}-\frac{2 \gamma_{j} \zeta(\alpha)}{\ell^{r} n-1}\right) .
$$

It is clear that $b_{2}>\hat{b}_{2}$. Thus we can prove that $b_{1}+b_{2}>c$ by proving that $b_{1}+\hat{b}_{2}-c>$ 0 . Using the result that

$$
\prod_{j=1}^{d}\left(\beta_{j}+a_{j}\right)=\sum_{\mathfrak{u} \subseteq \mathcal{D}}\left(\prod_{j \notin \mathfrak{u}} \beta_{j} \prod_{j \in \mathfrak{u}} a_{j}\right)=\prod_{j=1}^{d} \beta_{j}+\sum_{\emptyset \neq \mathfrak{u} \subseteq \mathcal{D}}\left(\prod_{j \notin \mathfrak{u}} \beta_{j} \prod_{j \in \mathfrak{u}} a_{j}\right),
$$

where $\mathcal{D}=\{1,2, \ldots, d\}$, we have

$$
\begin{aligned}
b_{1}+\hat{b}_{2}-c & =\prod_{j=1}^{d}\left(\beta_{j}+2 \gamma_{j} \zeta(\alpha)\right)+\left(\ell^{r} n-1\right) \prod_{j=1}^{d}\left(\beta_{j}-\frac{2 \gamma_{j} \zeta(\alpha)}{\ell^{r} n-1}\right)-\ell^{r} n \prod_{j=1}^{d} \beta_{j} \\
& =\sum_{\emptyset \neq \mathfrak{u} \subseteq \mathcal{D}}\left(\prod_{j \notin \mathfrak{u}} \beta_{j} \prod_{j \in \mathfrak{u}}\left(2 \gamma_{j} \zeta(\alpha)\right)+\left(\ell^{r} n-1\right) \sum_{\emptyset \neq \mathfrak{u} \subseteq \mathcal{D}}\left(\prod_{j \notin \mathfrak{u}} \beta_{j} \prod_{j \in \mathfrak{u}}\left(-\frac{2 \gamma_{j} \zeta(\alpha)}{\ell^{r} n-1}\right)\right)\right. \\
& =\sum_{\emptyset \neq \mathfrak{u} \subseteq \mathcal{D}}\left(S(\mathfrak{u}) \prod_{j \notin \mathfrak{u}} \beta_{j} \prod_{j \in \mathfrak{u}}\left(2 \gamma_{j} \zeta(\alpha)\right)\right),
\end{aligned}
$$


where

$$
S(\mathfrak{u})=1+\left(\ell^{r} n-1\right)\left(-\frac{1}{\ell^{r} n-1}\right)^{|\mathfrak{u}|} .
$$

Clearly $S(\mathfrak{u})>0$ if $|\mathfrak{u}|$ is even. For $|\mathfrak{u}| \geq 1$ odd, we have

$$
S(\mathfrak{u})=1-\left(\ell^{r} n-1\right)^{1-|\mathfrak{u}|} \geq 1-1=0 .
$$

Thus we conclude that $b_{1}+\hat{b}_{2}-c>0$ and hence $b_{1}+b_{2}>c$.

In the previous theorem, we made the assumption that $\rho_{\ell, r}<1$ and that (2.7) was true. Attempts to prove that (2.7) is always true have not been successful. However, all our numerical test calculations with $\ell=2, \alpha=2, \beta_{j}=1$, and various choices of $\gamma_{j}$ indicate that (2.7) does at least hold for this set of parameters. For other sets of parameters, readers will need to be content with doing their own calculations to see whether it holds or not for their particular situation.

The next result gives some sufficient conditions for $\rho_{2, r}$ to be less than one.

Lemma 2.4. Let $\rho_{\ell, r}$ be defined as in Theorem 2.3, and set $\ell=2$. If $\alpha \geq 2$ and

$$
\frac{\gamma_{r}}{\beta_{r}}>\frac{1}{\left(2-2^{2-\alpha}\right) \zeta(\alpha)}
$$

then $\rho_{2, r}<1$.

Proof. A product of positive terms is guaranteed to be less than one when each of the terms is less than one. From the definition of $\rho_{\ell, r}$, we see that if $\ell=2$, then this is the case when

$$
\frac{2 \beta_{j}+2^{2-\alpha} \gamma_{j} \zeta(\alpha)}{\beta_{j}+2 \gamma_{j} \zeta(\alpha)}<1
$$

for all $j=1,2, \ldots, r$. When rearranged, this yields

$$
\frac{\gamma_{j}}{\beta_{j}}>\frac{1}{\left(2-2^{2-\alpha}\right) \zeta(\alpha)} .
$$

Since the sequence $\left\{\frac{\gamma_{j}}{\beta_{j}}\right\}$ is nonincreasing, this completes the proof.

In the case when $\alpha=2$, the condition of the lemma becomes $\gamma_{r} / \beta_{r}>1 / \zeta(2)=$ $6 / \pi^{2} \approx 0.6079$. This suggests that when $\alpha=2$, it is worthwhile to take $r$ to be at least one when $\gamma_{1} / \beta_{1}>6 / \pi^{2}$.

In a sense, the quantity $\rho_{\ell, r}$ gives an indication of how much we can gain (or lose) by copying. Later in section 4 , we will see that though $\sqrt{\rho_{2, r}}$ is concerned with a ratio of means, the values of $\sqrt{\rho_{2, r}}$ nevertheless provide a measure of the ratios of the worst-case errors between intermediate-rank lattice rules and rank-1 lattice rules with approximately the same number of points.

2.3. Component-by-component construction. We now consider finding the components of the generating vector $\boldsymbol{z}$ one at a time. Keeping in mind the relationship of our intermediate-rank lattice rules with rank-1 lattice rules, we can construct $\overline{\boldsymbol{z}}$ for the rank-1 lattice rule with weights $\boldsymbol{\beta}$ and $\bar{\gamma}$ using the component-by-component Algorithm 8 of [9] from which we can then obtain the corresponding $\boldsymbol{z}$. This yields the same result as constructing $\boldsymbol{z}$ directly using Algorithm 2.5 below.

Algorithm 2.5. Given $1 \leq r \leq d$ and $n$ a prime number:

1. Set $z_{1}$, the first component of $\boldsymbol{z}$, to 1 . 
2. For $s=2,3, \ldots, r$, find $z_{s} \in \mathbb{Z}_{n}=\{1,2, \ldots, n-1\}$ such that

$$
e_{n, s, \operatorname{copy}(\ell, s)}^{2}\left(1, z_{2}, \ldots, z_{s}\right)=-\prod_{j=1}^{s} \beta_{j}+\frac{1}{n} \sum_{k=0}^{n-1} \prod_{j=1}^{s}\left(\beta_{j}+\frac{\gamma_{j}}{\ell^{\alpha}} \sum_{h=-\infty}^{\infty} \frac{e^{2 \pi \mathrm{i} h \ell k z_{j} / n}}{|h|^{\alpha}}\right)
$$

is minimized.

3. For $s=r+1, r+2, \ldots, d$, find $z_{s} \in \mathbb{Z}_{n}$ such that

$$
\begin{aligned}
e_{n, s, \operatorname{copy}(\ell, r)}^{2}\left(1, z_{2}, \ldots, z_{s}\right) & \\
=-\prod_{j=1}^{s} \beta_{j}+\frac{1}{n} \sum_{k=0}^{n-1}[ & \prod_{j=1}^{r}\left(\beta_{j}+\frac{\gamma_{j}}{\ell^{\alpha}} \sum_{h=-\infty}^{\infty} \frac{e^{2 \pi \mathrm{i} h \ell k z_{j} / n}}{|h|^{\alpha}}\right) \\
& \left.\times \prod_{j=r+1}^{d}\left(\beta_{j}+\gamma_{j} \sum_{h=-\infty}^{\infty} \frac{e^{2 \pi \mathrm{i} h k z_{j} / n}}{|h|^{\alpha}}\right)\right]
\end{aligned}
$$

is minimized.

Theorem 1 and Corollary 2 in [9] give the theoretical foundation behind such a construction for rank-1 lattice rules. We present the corresponding results here for intermediate-rank lattice rules. Note that Theorem 2.6 below is a slight improvement over the corresponding Theorem 1 of [9]. The proof is thus included in the appendix for completeness. (Such an improvement for rank-1 lattice rules was first obtained in [1] by using a different argument.)

THEOREM 2.6. Let $\boldsymbol{z}=\left(1, z_{2}, \ldots, z_{d}\right)$ be constructed component-by-component as in Algorithm 2.5.

(a) For each $s=1,2, \ldots, r$, we have

$$
e_{n, s, \operatorname{copy}(\ell, s)}^{2}\left(1, z_{2}, \ldots, z_{s}\right) \leq(n-1)^{-\frac{1}{\lambda}} \prod_{j=1}^{s}\left(\beta_{j}^{\lambda}+\frac{2 \gamma_{j}^{\lambda} \zeta(\alpha \lambda)}{\ell^{\alpha \lambda}}\right)^{\frac{1}{\lambda}}
$$

for all $\lambda$ satisfying $\frac{1}{\alpha}<\lambda \leq 1$.

(b) For each $s=r+1, r+2, \ldots, d$, we have

$$
\begin{aligned}
& e_{n, s, \operatorname{copy}(\ell, r)}^{2}\left(1, z_{2}, \ldots, z_{s}\right) \\
& \leq(n-1)^{-\frac{1}{\lambda}} \prod_{j=1}^{r}\left(\beta_{j}^{\lambda}+\frac{2 \gamma_{j}^{\lambda} \zeta(\alpha \lambda)}{\ell^{\alpha \lambda}}\right)^{\frac{1}{\lambda}} \prod_{j=r+1}^{s}\left(\beta_{j}^{\lambda}+2 \gamma_{j}^{\lambda} \zeta(\alpha \lambda)\right)^{\frac{1}{\lambda}}
\end{aligned}
$$

for all $\lambda$ satisfying $\frac{1}{\alpha}<\lambda \leq 1$.

It can be shown from the bounds above that the intermediate-rank lattice rules constructed using Algorithm 2.5 satisfy strong QMC tractability error bounds and achieve the optimal rate of convergence.

TheOREM 2.7. For fixed $r$ satisfying $1 \leq r \leq d$ and $n$ a prime number, let $N=$ $\ell^{r} n$ denote the total number of quadrature points, and let $\boldsymbol{z}$ be constructed componentby-component as in Algorithm 2.5. Then this $\boldsymbol{z}$ satisfies

$$
e_{n, d, \operatorname{copy}(\ell, r)}(\boldsymbol{z}) \leq C_{d}(\delta) N^{-\frac{\alpha}{2}+\delta} e_{0, d} \quad \text { for all } \quad 0<\delta \leq \frac{\alpha-1}{2},
$$


where

$$
\begin{aligned}
C_{d}(\delta)=\left(2 \ell^{r}\right)^{\frac{\alpha}{2}-\delta} & \prod_{j=1}^{r}\left[1+2 \ell^{-\frac{\alpha}{\alpha-2 \delta}}\left(\frac{\gamma_{j}}{\beta_{j}}\right)^{\frac{1}{\alpha-2 \delta}} \zeta\left(\frac{\alpha}{\alpha-2 \delta}\right)\right]^{\frac{\alpha}{2}-\delta} \\
& \times \prod_{j=r+1}^{d}\left[1+2\left(\frac{\gamma_{j}}{\beta_{j}}\right)^{\frac{1}{\alpha-2 \delta}} \zeta\left(\frac{\alpha}{\alpha-2 \delta}\right)\right]^{\frac{\alpha}{2}-\delta}
\end{aligned}
$$

is independent of $N$. Moreover, if

$$
\sum_{j=r+1}^{\infty}\left(\frac{\gamma_{j}}{\beta_{j}}\right)^{\frac{1}{\alpha-2 \delta}}<\infty
$$

then

$$
C_{d}(\delta) \leq C_{\infty}(\delta)<\infty
$$

that is, $e_{n, d, \operatorname{copy}(\ell, r)}(\boldsymbol{z})$ is $O\left(N^{-\alpha / 2+\delta}\right)$ for $\delta>0$, independently of $d$.

Proof. It follows from Theorem 2.6 that the $\boldsymbol{z}$ constructed by Algorithm 2.5 satisfies

$$
\begin{aligned}
& e_{n, d, \operatorname{copy}(\ell, r)}(\boldsymbol{z}) \\
& \leq(n-1)^{-\frac{1}{2 \lambda}} \prod_{j=1}^{r}\left(\beta_{j}^{\lambda}+\frac{2 \gamma_{j}^{\lambda} \zeta(\alpha \lambda)}{\ell^{\alpha \lambda}}\right)^{\frac{1}{2 \lambda}} \prod_{j=r+1}^{d}\left(\beta_{j}^{\lambda}+2 \gamma_{j}^{\lambda} \zeta(\alpha \lambda)\right)^{\frac{1}{2 \lambda}} \\
& \leq\left(\frac{n \ell^{r}}{2 \ell^{r}}\right)^{-\frac{1}{2 \lambda}} \prod_{j=1}^{r}\left(1+2 \ell^{-\alpha \lambda}\left(\frac{\gamma_{j}}{\beta_{j}}\right)^{\lambda} \zeta(\alpha \lambda)\right)^{\frac{1}{2 \lambda}} \prod_{j=r+1}^{d}\left(1+2\left(\frac{\gamma_{j}}{\beta_{j}}\right)^{\lambda} \zeta(\alpha \lambda)\right)^{\frac{1}{2 \lambda}} \prod_{j=1}^{d} \beta_{j}^{\frac{1}{2}}
\end{aligned}
$$

for all $\frac{1}{\alpha}<\lambda \leq 1$. Now with the substitution of

$$
-\frac{\alpha}{2}+\delta=-\frac{1}{2 \lambda}
$$

the condition $\frac{1}{\alpha}<\lambda \leq 1$ becomes $0<\delta \leq \frac{\alpha-1}{2}$, and we obtain

$$
e_{n, d, \operatorname{copy}(\ell, r)}(\boldsymbol{z}) \leq C_{d}(\delta) N^{-\frac{\alpha}{2}+\delta} e_{0, d} \quad \text { for all } 0<\delta \leq \frac{\alpha-1}{2},
$$

where

$$
\begin{aligned}
C_{d}(\delta)=\left(2 \ell^{r}\right)^{\frac{\alpha}{2}-\delta} & \prod_{j=1}^{r}\left[1+2 \ell^{-\frac{\alpha}{\alpha-2 \delta}}\left(\frac{\gamma_{j}}{\beta_{j}}\right)^{\frac{1}{\alpha-2 \delta}} \zeta\left(\frac{\alpha}{\alpha-2 \delta}\right)\right]^{\frac{\alpha}{2}-\delta} \\
& \times \prod_{j=r+1}^{d}\left[1+2\left(\frac{\gamma_{j}}{\beta_{j}}\right)^{\frac{1}{\alpha-2 \delta}} \zeta\left(\frac{\alpha}{\alpha-2 \delta}\right)\right]^{\frac{\alpha}{2}-\delta} \leq C_{\infty}(\delta),
\end{aligned}
$$


and

$$
\begin{aligned}
C_{\infty}(\delta)=\left(2 \ell^{r}\right)^{\frac{\alpha}{2}-\delta} \prod_{j=1}^{r}\left[1+2 \ell^{-\frac{\alpha}{\alpha-2 \delta}}\left(\frac{\gamma_{j}}{\beta_{j}}\right)^{\frac{1}{\alpha-2 \delta}} \zeta\left(\frac{\alpha}{\alpha-2 \delta}\right)\right]^{\frac{\alpha}{2}-\delta} \\
\quad \times \exp \left(\left(\frac{\alpha}{2}-\delta\right) \sum_{j=r+1}^{\infty} \log \left(1+2\left(\frac{\gamma_{j}}{\beta_{j}}\right)^{\frac{1}{\alpha-2 \delta}} \zeta\left(\frac{\alpha}{\alpha-2 \delta}\right)\right)\right) \\
\leq\left(2 \ell^{r}\right)^{\frac{\alpha}{2}-\delta} \prod_{j=1}^{r}\left[1+2 \ell^{-\frac{\alpha}{\alpha-2 \delta}}\left(\frac{\gamma_{j}}{\beta_{j}}\right)^{\frac{1}{\alpha-2 \delta}} \zeta\left(\frac{\alpha}{\alpha-2 \delta}\right)\right]^{\frac{\alpha}{2}-\delta} \\
\quad \times \exp \left((\alpha-2 \delta) \zeta\left(\frac{\alpha}{\alpha-2 \delta}\right) \sum_{j=r+1}^{\infty}\left(\frac{\gamma_{j}}{\beta_{j}}\right)^{\frac{1}{\alpha-2 \delta}}\right),
\end{aligned}
$$

where we have used the fact that $\log (1+x) \leq x$ for $x \geq 0$. It is clear from this expression that for $\delta>0, C_{\infty}(\delta)<\infty$ if

$$
\sum_{j=r+1}^{\infty}\left(\frac{\gamma_{j}}{\beta_{j}}\right)^{\frac{1}{\alpha-2 \delta}}<\infty
$$

This completes the proof.

3. Shifted intermediate-rank lattice rules in weighted Sobolev spaces. Now we change the function spaces to weighted Sobolev spaces considered in [10]. These spaces are also parameterized by two sequences of positive weights $\boldsymbol{\beta}$ and $\boldsymbol{\gamma}$ satisfying

$$
\frac{\gamma_{1}}{\beta_{1}} \geq \frac{\gamma_{2}}{\beta_{2}} \geq \cdots .
$$

The inner product in these spaces is given by

$$
\langle f, g\rangle_{d}:=\sum_{\mathfrak{u} \subseteq\{1,2, \ldots, d\}}\left(\prod_{j \notin \mathfrak{u}} \beta_{j}^{-1} \prod_{j \in \mathfrak{u}} \gamma_{j}^{-1} \int_{[0,1]^{|\mathfrak{u}|}} \frac{\partial^{|\mathfrak{u}|}}{\partial \boldsymbol{x}_{\mathfrak{u}}} f\left(\boldsymbol{x}_{\mathfrak{u}}, \mathbf{1}\right) \frac{\partial^{|\mathfrak{u}|}}{\partial \boldsymbol{x}_{\mathfrak{u}}} g\left(\boldsymbol{x}_{\mathfrak{u}}, \mathbf{1}\right) \mathrm{d} \boldsymbol{x}_{\mathfrak{u}}\right),
$$

where $\left(\boldsymbol{x}_{\mathfrak{u}}, \mathbf{1}\right)$ is a $d$-dimensional vector whose $j$ th component is $x_{j}$ if $j \in \mathfrak{u}$ and 1 if $j \notin \mathfrak{u}$. Similar spaces have been considered previously (for example, see [12], [13], and $[16])$. The worst-case error for a QMC rule (1.1) in these spaces is given by

$$
\begin{aligned}
e_{n, d}^{2}\left(\boldsymbol{x}_{0}, \ldots, \boldsymbol{x}_{n-1}\right)= & \prod_{j=1}^{d}\left(\beta_{j}+\frac{\gamma_{j}}{3}\right)-\frac{2}{n} \sum_{i=0}^{n-1} \prod_{j=1}^{d}\left(\beta_{j}+\frac{\gamma_{j}}{2}\left(1-x_{i, j}^{2}\right)\right) \\
& +\frac{1}{n^{2}} \sum_{i=0}^{n-1} \sum_{k=0}^{n-1} \prod_{j=1}^{d}\left(\beta_{j}+\gamma_{j}\left[1-\max \left(x_{i, j}, x_{k, j}\right)\right]\right),
\end{aligned}
$$

and the initial error is

$$
e_{0, d}=\prod_{j=1}^{d}\left(\beta_{j}+\frac{\gamma_{j}}{3}\right)^{\frac{1}{2}}
$$


Similar to the weighted Korobov spaces, it can be shown that if the weights satisfy (2.2), then an upper bound for the square worst-case error of the form (2.3) is enough to ensure strong QMC tractability in weighted Sobolev spaces.

We now consider the $\boldsymbol{\Delta}$-shift of the $(\ell, r)$-copy of a rank-1 lattice rule with generating vector $\boldsymbol{z}$, that is, a rule with points given by

$$
\left\{\left\{\frac{i \boldsymbol{z}}{n}+\frac{\left(m_{1}, \ldots, m_{r}, 0, \ldots, 0\right)}{\ell}+\boldsymbol{\Delta}\right\}: 0 \leq i \leq n-1,0 \leq m_{1}, \ldots, m_{r} \leq \ell-1\right\},
$$

where $\ell \geq 1, \operatorname{gcd}(\ell, n)=1$, and $0 \leq r \leq d$. Let $e_{n, d, \operatorname{copy}(\ell, r)}(\boldsymbol{z}, \boldsymbol{\Delta})$ denote the worstcase error for such a rule. An expression for $e_{n, d, \operatorname{copy}(\ell, r)}^{2}(\boldsymbol{z}, \boldsymbol{\Delta})$ can be derived from (3.1).

Here we give just the general ideas of the existence and the construction of a good shifted intermediate-rank lattice rule. The full details follow closely the arguments from [12] and [13].

To obtain an upper bound on the square worst-case error, we define the mean of $e_{n, d, \operatorname{copy}(\ell, r)}^{2}(\boldsymbol{z}, \boldsymbol{\Delta})$ over all values of $\boldsymbol{z} \in \mathbb{Z}_{n}^{d}$ and $\boldsymbol{\Delta} \in[0,1]^{d}$ by

$$
M_{n, d, \operatorname{copy}(\ell, r)}:=\frac{1}{(n-1)^{d}} \sum_{\boldsymbol{z} \in \mathbb{Z}_{n}^{d}}\left(\int_{[0,1]^{d}} e_{n, d, \operatorname{copy}(\ell, r)}^{2}(\boldsymbol{z}, \boldsymbol{\Delta}) \mathrm{d} \boldsymbol{\Delta}\right) .
$$

Using a known relationship between weighted Korobov spaces and weighted Sobolev spaces (see [5]), we see that this mean is exactly the mean given in Theorem 2.2 with $\alpha$ replaced by $2, \beta_{j}$ replaced by $\beta_{j}+\frac{\gamma_{j}}{3}$, and $\gamma_{j}$ replaced by $\frac{\gamma_{j}}{2 \pi^{2}}$. An upper bound for $M_{n, d, \operatorname{copy}(\ell, r)}$ follows in the same way from Theorem 2.2:

$$
M_{n, d, \operatorname{copy}(\ell, r)} \leq \frac{1}{n} \prod_{j=1}^{d}\left(\beta_{j}+\frac{\gamma_{j}}{3}+\frac{2 \gamma_{j}}{2 \pi^{2}} \zeta(2)\right)=\frac{1}{n} \prod_{j=1}^{d}\left(\beta_{j}+\frac{\gamma_{j}}{2}\right) .
$$

We thus conclude that there exists at least one pair $(\boldsymbol{z}, \boldsymbol{\Delta})$ such that $e_{n, d, \operatorname{copy}(\ell, r)}^{2}(\boldsymbol{z}, \boldsymbol{\Delta})$ is bounded by this upper bound on the mean. Since this bound is of the form (2.3), we conclude that shifted intermediate-rank lattice rules achieve strong QMC tractability error bounds in weighted Sobolev spaces.

Let $e_{n, d+1, \ell}\left(\boldsymbol{x}_{0}, \ldots, \boldsymbol{x}_{n-1} ; z_{d+1}, \Delta_{d+1}\right)$ denote the worst-case error for a QMC rule with the set of points

$$
\left\{\left(\boldsymbol{x}_{i},\left\{\frac{i z_{d+1}}{n}+\frac{m}{\ell}+\Delta_{d+1}\right\}\right): 0 \leq i \leq n-1,0 \leq m \leq \ell-1\right\} .
$$

These are $(d+1)$-dimensional points obtained by appending $\left\{\frac{i z_{d+1}}{n}+\frac{m}{\ell}+\Delta_{d+1}\right\}$ to the existing $d$ components of $\boldsymbol{x}_{i}$. To construct the pair $\left(z_{d+1}, \Delta_{d+1}\right)$ component-bycomponent, we define the following mean:

$$
m_{n, d+1, \ell}\left(\boldsymbol{x}_{0}, \ldots, \boldsymbol{x}_{n-1} ; z_{d+1}\right):=\int_{0}^{1} e_{n, d+1, \ell}^{2}\left(\boldsymbol{x}_{0}, \ldots, \boldsymbol{x}_{n-1} ; z_{d+1}, \Delta_{d+1}\right) \mathrm{d} \Delta_{d+1} .
$$

Let us assume that the points $\boldsymbol{x}_{0}, \ldots, \boldsymbol{x}_{n-1}$ satisfy

$$
e_{n, d}^{2}\left(\boldsymbol{x}_{0}, \ldots, \boldsymbol{x}_{n-1}\right) \leq \frac{1}{n} \prod_{j=1}^{d}\left(\beta_{j}+\gamma_{j}\right) .
$$


Suppose we choose $z_{d+1}$ from the set $\mathbb{Z}_{n}$ to minimize $m_{n, d+1, \ell}\left(\boldsymbol{x}_{0}, \ldots, \boldsymbol{x}_{n-1} ; z_{d+1}\right)$ and then choose $\Delta_{d+1}$ from the set $\left\{\frac{2 m-1}{2 n}: 1 \leq m \leq n-1\right\}$ so that the square worstcase error $e_{n, d+1, \ell}^{2}\left(\boldsymbol{x}_{0}, \ldots, \boldsymbol{x}_{n-1} ; z_{d+1}, \Delta_{d+1}\right)$ is minimized. Then by using involved algebraic manipulations and the arguments from [12], these choices of $z_{d+1}$ and $\Delta_{d+1}$ can be shown to satisfy

$$
e_{n, d+1, \ell}^{2}\left(\boldsymbol{x}_{0}, \ldots, \boldsymbol{x}_{n-1} ; z_{d+1}, \Delta_{d+1}\right) \leq \frac{1}{n} \prod_{j=1}^{d+1}\left(\beta_{j}+\gamma_{j}\right) .
$$

Note that the result also holds for $\ell=1$; that is, there is no "copying" in the $(d+1)$ th dimension. For $d=1$, we can show that there exists $\left(z_{1}, \Delta_{1}\right)$ satisfying

$$
e_{n, 1, \operatorname{copy}(\ell, 1)}^{2}\left(z_{1}, \Delta_{1}\right) \leq \frac{1}{n}\left(\beta_{1}+\gamma_{1}\right) .
$$

All of the above leads us to the following algorithm for constructing a pair $(\boldsymbol{z}, \boldsymbol{\Delta})$ such that for all $s=1, \ldots, d$,

$$
e_{n, s, \operatorname{copy}(\ell, \min (s, r))}^{2}\left(\left(z_{1}, \ldots, z_{s}\right),\left(\Delta_{1}, \ldots, \Delta_{s}\right)\right) \leq \frac{1}{n} \prod_{j=1}^{s}\left(\beta_{j}+\gamma_{j}\right) .
$$

In the following algorithm, the notation

$$
m_{n, s, \operatorname{copy}(\ell, r)}\left(\left(1, z_{2}, \ldots, z_{s-1}\right),\left(\Delta_{1}, \Delta_{2}, \ldots, \Delta_{s-1}\right) ; z_{s}\right)
$$

is used to denote the quantity $m_{n, s, \ell}\left(\boldsymbol{x}_{0}, \ldots, \boldsymbol{x}_{n-1} ; z_{s}\right)$ in the situation when $\boldsymbol{x}_{0}, \ldots$, $\boldsymbol{x}_{n-1}$ are the points from an $(\ell, r)$-copy of an $(s-1)$-dimensional rank-1 lattice rule.

Algorithm 3.1. Given $n$ a prime number and $1 \leq r \leq d$ :

1. Set $z_{1}$, the first component of $\boldsymbol{z}$, to 1 .

2. Find $\Delta_{1} \in\left\{\frac{1}{2 n}, \frac{3}{2 n}, \ldots, \frac{2 n-1}{2 n}\right\}$ to minimize $e_{n, 1, \operatorname{copy}(\ell, 1)}^{2}\left(1, \Delta_{1}\right)$.

3. For $s=2,3, \ldots, r$, do the following:

(a) Find $z_{s} \in\{1,2, \ldots, n-1\}$ to minimize

$$
m_{n, s, \operatorname{copy}(\ell, s)}\left(\left(1, z_{2}, \ldots, z_{s-1}\right),\left(\Delta_{1}, \Delta_{2}, \ldots, \Delta_{s-1}\right) ; z_{s}\right) .
$$

(b) Find $\Delta_{s} \in\left\{\frac{1}{2 n}, \frac{3}{2 n}, \ldots, \frac{2 n-1}{2 n}\right\}$ to minimize

$$
e_{n, s, \operatorname{copy}(\ell, s)}^{2}\left(\left(1, z_{2}, \ldots, z_{s}\right),\left(\Delta_{1}, \Delta_{2}, \ldots, \Delta_{s}\right)\right) \text {. }
$$

4. For $s=r+1, r+2, \ldots, d$, do the following:

(a) Find $z_{s} \in\{1,2, \ldots, n-1\}$ to minimize

$$
m_{n, s, \operatorname{copy}(\ell, r)}\left(\left(1, z_{2}, \ldots, z_{s-1}\right),\left(\Delta_{1}, \Delta_{2}, \ldots, \Delta_{s-1}\right) ; z_{s}\right) .
$$

(b) Find $\Delta_{s} \in\left\{\frac{1}{2 n}, \frac{3}{2 n}, \ldots, \frac{2 n-1}{2 n}\right\}$ to minimize

$$
e_{n, s, \operatorname{copy}(\ell, r)}^{2}\left(\left(1, z_{2}, \ldots, z_{s}\right),\left(\Delta_{1}, \Delta_{2}, \ldots, \Delta_{s}\right)\right) .
$$

The cost for the construction is $O\left(n^{3} d^{2}\right)$ operations, and it is dominated by the construction of the shift. In [13] the idea of using a number of random shifts was introduced. This not only cuts the cost of the construction down to $O\left(n^{2} d^{2}\right)$ operations; it also allows error estimation. The reference [3] contains detailed discussions 
on randomized QMC methods. Following [13], we can construct the generating vector component-by-component by minimizing over the mean

$$
F_{n, d, \operatorname{copy}(\ell, r)}(\boldsymbol{z}):=\int_{[0,1]^{d}} e_{n, d, \operatorname{copy}(\ell, r)}^{2}(\boldsymbol{z}, \boldsymbol{\Delta}) \mathrm{d} \boldsymbol{\Delta} .
$$

Algorithm 3.2. Given $n$ a prime number and $1 \leq r \leq d$ :

1. Set $z_{1}$, the first component of $\boldsymbol{z}$, to 1 .

2. For $s=2,3, \ldots, r$, find $z_{s} \in\{1,2, \ldots, n-1\}$ to minimize

$$
F_{n, s, \operatorname{copy}(\ell, s)}\left(1, z_{2}, \ldots, z_{s}\right) \text {. }
$$

3. For $s=r+1, r+2, \ldots, d$, find $z_{s} \in\{1,2, \ldots, n-1\}$ to minimize

$$
F_{n, s, \operatorname{copy}(\ell, r)}\left(1, z_{2}, \ldots, z_{s}\right) \text {. }
$$

Using again the relationship between weighted Korobov spaces and weighted Sobolev spaces, we can obtain the corresponding value of the quantity $\rho_{\ell, r}$ given in Theorem 2.3 for weighted Sobolev spaces by replacing $\alpha$ with $2, \beta_{j}$ with $\beta_{j}+\frac{\gamma_{j}}{3}$, and $\gamma_{j}$ with $\frac{\gamma_{j}}{2 \pi^{2}}$. This yields

$$
\rho_{\ell, r}=\prod_{j=1}^{r} \frac{\ell \beta_{j}+\gamma_{j}\left(\frac{\ell}{3}+\frac{1}{6 \ell}\right)}{\beta_{j}+\frac{\gamma_{j}}{2}},
$$

which is greater than 1 for all $\ell \geq 2$. This means that it is unlikely for the ratio $R_{n, d, \ell, r}$ to be less than 1 , and thus copying may not give better results in weighted Sobolev spaces.

4. Numerical results. We will consider weighted Korobov spaces with $\alpha=2$. In this case, the square worst-case error can be written as

$$
\begin{aligned}
e_{n, d, \operatorname{copy}(\ell, r)}^{2}(\boldsymbol{z})=-\prod_{j=1}^{d} \beta_{j}+\frac{1}{n} \sum_{k=0}^{n-1} & {\left[\prod_{j=1}^{r}\left(\beta_{j}+\frac{2 \pi^{2} \gamma_{j}}{\ell^{2}} B_{2}\left(\left\{\frac{\ell k z_{j}}{n}\right\}\right)\right)\right.} \\
& \left.\times \prod_{j=r+1}^{d}\left(\beta_{j}+2 \pi^{2} \gamma_{j} B_{2}\left(\left\{\frac{k z_{j}}{n}\right\}\right)\right)\right],
\end{aligned}
$$

where for $x \in[0,1], B_{2}(x)=x^{2}-x+1 / 6$ is the Bernoulli polynomial of degree 2 . In the implementation of Steps 2 and 3 of Algorithm 2.5, we will consider only values of $z_{s}$ in $\{1,2, \ldots,(n-1) / 2\}$, since

$$
B_{2}\left(\left\{\frac{k z_{s}}{n}\right\}\right)=B_{2}\left(\left\{\frac{k\left(n-z_{s}\right)}{n}\right\}\right) \text { and } B_{2}\left(\left\{\frac{\ell k z_{s}}{n}\right\}\right)=B_{2}\left(\left\{\frac{\ell k\left(n-z_{s}\right)}{n}\right\}\right) .
$$

For $\alpha=2, \boldsymbol{\beta}=\mathbf{1}$, and two different sequences of $\boldsymbol{\gamma}$,

$$
\gamma_{j}=0.9^{j} \quad \text { and } \quad \gamma_{j}=\frac{1}{j^{2}}
$$

we want to see if intermediate-rank lattice rules are better than rank-1 lattice rules with approximately the same number of points. More precisely, when $\ell=2$, we 
TABLE 4.1

Total number of points close to $4000, \gamma_{j}=0.9^{j}$.

\begin{tabular}{|r|c|cc|cc|cc|}
\hline$d$ & 4001 & $\begin{array}{c}2003 \times 2^{1} \\
=4006\end{array}$ & $\begin{array}{c}1999 \times 2^{1} \\
=3998\end{array}$ & $\begin{array}{c}1009 \times 2^{2} \\
=4036\end{array}$ & $\begin{array}{c}997 \times 2^{2} \\
=3988\end{array}$ & $\begin{array}{c}503 \times 2^{3} \\
=4024\end{array}$ & $\begin{array}{c}499 \times 2^{3} \\
=3992\end{array}$ \\
\hline 10 & $2.9726 \mathrm{e}+00$ & $2.8068 \mathrm{e}+00$ & $2.8005 \mathrm{e}+00$ & $2.6666 \mathrm{e}+00$ & $2.6874 \mathrm{e}+00$ & $2.5965 \mathrm{e}+00$ & $2.6068 \mathrm{e}+00$ \\
20 & $3.8737 \mathrm{e}+01$ & $3.6466 \mathrm{e}+01$ & $3.6455 \mathrm{e}+01$ & $3.4687 \mathrm{e}+01$ & $3.4922 \mathrm{e}+01$ & $3.3752 \mathrm{e}+01$ & $3.3887 \mathrm{e}+01$ \\
30 & $1.1022 \mathrm{e}+02$ & $1.0374 \mathrm{e}+02$ & $1.0372 \mathrm{e}+02$ & $9.8675 \mathrm{e}+01$ & $9.9337 \mathrm{e}+01$ & $9.6009 \mathrm{e}+01$ & $9.6392 \mathrm{e}+01$ \\
40 & $1.6309 \mathrm{e}+02$ & $1.5348 \mathrm{e}+02$ & $1.5346 \mathrm{e}+02$ & $1.4598 \mathrm{e}+02$ & $1.4696 \mathrm{e}+02$ & $1.4204 \mathrm{e}+02$ & $1.4260 \mathrm{e}+02$ \\
50 & $1.8768 \mathrm{e}+02$ & $1.7661 \mathrm{e}+02$ & $1.7659 \mathrm{e}+02$ & $1.6798 \mathrm{e}+02$ & $1.6911 \mathrm{e}+02$ & $1.6344 \mathrm{e}+02$ & $1.6409 \mathrm{e}+02$ \\
60 & $1.9719 \mathrm{e}+02$ & $1.8556 \mathrm{e}+02$ & $1.8554 \mathrm{e}+02$ & $1.7650 \mathrm{e}+02$ & $1.7768 \mathrm{e}+02$ & $1.7172 \mathrm{e}+02$ & $1.7241 \mathrm{e}+02$ \\
70 & $2.0063 \mathrm{e}+02$ & $1.8879 \mathrm{e}+02$ & $1.8878 \mathrm{e}+02$ & $1.7957 \mathrm{e}+02$ & $1.8077 \mathrm{e}+02$ & $1.7472 \mathrm{e}+02$ & $1.7542 \mathrm{e}+02$ \\
80 & $2.0185 \mathrm{e}+02$ & $1.8994 \mathrm{e}+02$ & $1.8992 \mathrm{e}+02$ & $1.8066 \mathrm{e}+02$ & $1.8187 \mathrm{e}+02$ & $1.7578 \mathrm{e}+02$ & $1.7648 \mathrm{e}+02$ \\
90 & $2.0227 \mathrm{e}+02$ & $1.9034 \mathrm{e}+02$ & $1.9032 \mathrm{e}+02$ & $1.8104 \mathrm{e}+02$ & $1.8225 \mathrm{e}+02$ & $1.7615 \mathrm{e}+02$ & $1.7685 \mathrm{e}+02$ \\
100 & $2.0242 \mathrm{e}+02$ & $1.9048 \mathrm{e}+02$ & $1.9046 \mathrm{e}+02$ & $1.8118 \mathrm{e}+02$ & $1.8239 \mathrm{e}+02$ & $1.7628 \mathrm{e}+02$ & $1.7698 \mathrm{e}+02$ \\
\hline
\end{tabular}

TABLE 4.2

Total number of points close to $16000, \gamma_{j}=0.9^{j}$.

\begin{tabular}{|r|c|cc|cc|cc|}
\hline$d$ & 16007 & $\begin{array}{c}8009 \times 2^{1} \\
=16018\end{array}$ & $\begin{array}{c}7993 \times 2^{1} \\
=15986\end{array}$ & $\begin{array}{c}4003 \times 2^{2} \\
=16012\end{array}$ & $\begin{array}{c}4001 \times 2^{2} \\
=16004\end{array}$ & $\begin{array}{c}2003 \times 2^{3} \\
=16024\end{array}$ & $\begin{array}{c}1999 \times 2^{3} \\
=15992\end{array}$ \\
\hline 10 & $1.4365 \mathrm{e}+00$ & $1.3566 \mathrm{e}+00$ & $1.3606 \mathrm{e}+00$ & $1.2982 \mathrm{e}+00$ & $1.2973 \mathrm{e}+00$ & $1.2623 \mathrm{e}+00$ & $1.2621 \mathrm{e}+00$ \\
20 & $1.9268 \mathrm{e}+01$ & $1.8198 \mathrm{e}+01$ & $1.8231 \mathrm{e}+01$ & $1.7400 \mathrm{e}+01$ & $1.7413 \mathrm{e}+01$ & $1.6905 \mathrm{e}+01$ & $1.6922 \mathrm{e}+01$ \\
30 & $5.4841 \mathrm{e}+01$ & $5.1793 \mathrm{e}+01$ & $5.1887 \mathrm{e}+01$ & $4.9516 \mathrm{e}+01$ & $4.9554 \mathrm{e}+01$ & $4.8109 \mathrm{e}+01$ & $4.8157 \mathrm{e}+01$ \\
40 & $8.1141 \mathrm{e}+01$ & $7.6628 \mathrm{e}+01$ & $7.6767 \mathrm{e}+01$ & $7.3258 \mathrm{e}+01$ & $7.3314 \mathrm{e}+01$ & $7.1176 \mathrm{e}+01$ & $7.1247 \mathrm{e}+01$ \\
50 & $9.3371 \mathrm{e}+01$ & $8.8176 \mathrm{e}+01$ & $8.8337 \mathrm{e}+01$ & $8.4298 \mathrm{e}+01$ & $8.4363 \mathrm{e}+01$ & $8.1902 \mathrm{e}+01$ & $8.1984 \mathrm{e}+01$ \\
60 & $9.8103 \mathrm{e}+01$ & $9.2645 \mathrm{e}+01$ & $9.2813 \mathrm{e}+01$ & $8.8570 \mathrm{e}+01$ & $8.8638 \mathrm{e}+01$ & $8.6053 \mathrm{e}+01$ & $8.6138 \mathrm{e}+01$ \\
70 & $9.9815 \mathrm{e}+01$ & $9.4261 \mathrm{e}+01$ & $9.4433 \mathrm{e}+01$ & $9.0115 \mathrm{e}+01$ & $9.0184 \mathrm{e}+01$ & $8.7554 \mathrm{e}+01$ & $8.7641 \mathrm{e}+01$ \\
80 & $1.0042 \mathrm{e}+02$ & $9.4832 \mathrm{e}+01$ & $9.5004 \mathrm{e}+01$ & $9.0661 \mathrm{e}+01$ & $9.0730 \mathrm{e}+01$ & $8.8084 \mathrm{e}+01$ & $8.8172 \mathrm{e}+01$ \\
90 & $1.0063 \mathrm{e}+02$ & $9.5032 \mathrm{e}+01$ & $9.5205 \mathrm{e}+01$ & $9.0852 \mathrm{e}+01$ & $9.0922 \mathrm{e}+01$ & $8.8270 \mathrm{e}+01$ & $8.8358 \mathrm{e}+01$ \\
100 & $1.0070 \mathrm{e}+02$ & $9.5102 \mathrm{e}+01$ & $9.5275 \mathrm{e}+01$ & $9.0919 \mathrm{e}+01$ & $9.0988 \mathrm{e}+01$ & $8.8335 \mathrm{e}+01$ & $8.8423 \mathrm{e}+01$ \\
\hline
\end{tabular}

TABLE 4.3

Total number of points close to $64000, \gamma_{j}=0.9^{j}$.

\begin{tabular}{|r|c|cc|cc|cc|}
\hline$d$ & 64007 & $\begin{array}{c}32009 \times 2^{1} \\
=64018\end{array}$ & $\begin{array}{c}32003 \times 2^{1} \\
=64006\end{array}$ & $\begin{array}{c}16007 \times 2^{2} \\
=64028\end{array}$ & $\begin{array}{c}16001 \times 2^{2} \\
=64004\end{array}$ & $\begin{array}{c}8009 \times 2^{3} \\
=64072\end{array}$ & $\begin{array}{c}7993 \times 2^{3} \\
=63944\end{array}$ \\
\hline 10 & $6.8423 \mathrm{e}-01$ & $6.4784 \mathrm{e}-01$ & $6.4773 \mathrm{e}-01$ & $6.1683 \mathrm{e}-01$ & $6.1797 \mathrm{e}-01$ & $5.9937 \mathrm{e}-01$ & $6.0015 \mathrm{e}-01$ \\
20 & $9.6190 \mathrm{e}+00$ & $9.1124 \mathrm{e}+00$ & $9.0949 \mathrm{e}+00$ & $8.6927 \mathrm{e}+00$ & $8.6945 \mathrm{e}+00$ & $8.4445 \mathrm{e}+00$ & $8.4523 \mathrm{e}+00$ \\
30 & $2.7406 \mathrm{e}+01$ & $2.5958 \mathrm{e}+01$ & $2.5908 \mathrm{e}+01$ & $2.4763 \mathrm{e}+01$ & $2.4768 \mathrm{e}+01$ & $2.4055 \mathrm{e}+01$ & $2.4077 \mathrm{e}+01$ \\
40 & $4.0552 \mathrm{e}+01$ & $3.8408 \mathrm{e}+01$ & $3.8336 \mathrm{e}+01$ & $3.6640 \mathrm{e}+01$ & $3.6647 \mathrm{e}+01$ & $3.5592 \mathrm{e}+01$ & $3.5625 \mathrm{e}+01$ \\
50 & $4.6664 \mathrm{e}+01$ & $4.4197 \mathrm{e}+01$ & $4.4114 \mathrm{e}+01$ & $4.2162 \mathrm{e}+01$ & $4.2170 \mathrm{e}+01$ & $4.0956 \mathrm{e}+01$ & $4.0995 \mathrm{e}+01$ \\
60 & $4.9029 \mathrm{e}+01$ & $4.6436 \mathrm{e}+01$ & $4.6350 \mathrm{e}+01$ & $4.4299 \mathrm{e}+01$ & $4.4307 \mathrm{e}+01$ & $4.3032 \mathrm{e}+01$ & $4.3072 \mathrm{e}+01$ \\
70 & $4.9885 \mathrm{e}+01$ & $4.7247 \mathrm{e}+01$ & $4.7159 \mathrm{e}+01$ & $4.5072 \mathrm{e}+01$ & $4.5080 \mathrm{e}+01$ & $4.3783 \mathrm{e}+01$ & $4.3824 \mathrm{e}+01$ \\
80 & $5.0187 \mathrm{e}+01$ & $4.7533 \mathrm{e}+01$ & $4.7444 \mathrm{e}+01$ & $4.5345 \mathrm{e}+01$ & $4.5353 \mathrm{e}+01$ & $4.4048 \mathrm{e}+01$ & $4.4089 \mathrm{e}+01$ \\
90 & $5.0293 \mathrm{e}+01$ & $4.7633 \mathrm{e}+01$ & $4.7544 \mathrm{e}+01$ & $4.5441 \mathrm{e}+01$ & $4.5449 \mathrm{e}+01$ & $4.4141 \mathrm{e}+01$ & $4.4182 \mathrm{e}+01$ \\
100 & $5.0330 \mathrm{e}+01$ & $4.7668 \mathrm{e}+01$ & $4.7579 \mathrm{e}+01$ & $4.5474 \mathrm{e}+01$ & $4.5483 \mathrm{e}+01$ & $4.4173 \mathrm{e}+01$ & $4.4215 \mathrm{e}+01$ \\
\hline
\end{tabular}

want to know in how many dimensions to copy, that is, which value of $r=1,2$, or higher should we choose to get better rules than rank-1 lattice rules. We compare the worst-case errors for rules with approximately 4000, 16000, and 64000 points up to 100 dimensions. (Note that since $\boldsymbol{\beta}=\mathbf{1}$, the initial error $e_{0, d}$ is 1.) The results are presented in Tables 4.1 to 4.6. The second column of each of these tables contains the worst-case error for rank- 1 rules, while the other three columns contain the worst-case error for $r$ going from $r=1$ to $r=3$. To get a better picture of the results of copying, we divide the worst-case errors of intermediate-rank lattice rules at $d=100$ by those of rank-1 lattice rules with approximately the same number of points. These ratios are presented in Table 4.7.

We can see from the results that for $\gamma_{j}=0.9^{j}$, copying is good in at least the first three dimensions, but for $\gamma_{j}=1 / j^{2}$, it is only good to copy in the first dimension. This seems reasonable as in the first few dimensions the sequence $0.9,0.81,0.729, \ldots$ 
TABLE 4.4

Total number of points close to $4000, \gamma_{j}=1 / j^{2}$.

\begin{tabular}{|r|c|cc|cc|cc|}
\hline & & $\begin{array}{c}2003 \times 2^{1} \\
=4006\end{array}$ & $\begin{array}{c}1999 \times 2^{1} \\
=3998\end{array}$ & $\begin{array}{c}1009 \times 2^{2} \\
=4036\end{array}$ & $\begin{array}{c}997 \times 2^{2} \\
=3988\end{array}$ & $\begin{array}{c}503 \times 2^{3} \\
=4024\end{array}$ & $\begin{array}{c}499 \times 2^{3} \\
=3992\end{array}$ \\
\hline 10 & $1.9338 \mathrm{e}-02$ & $1.8362 \mathrm{e}-02$ & $1.8036 \mathrm{e}-02$ & $2.0006 \mathrm{e}-02$ & $2.0218 \mathrm{e}-02$ & $2.5298 \mathrm{e}-02$ & $2.5381 \mathrm{e}-02$ \\
20 & $2.5421 \mathrm{e}-02$ & $2.3923 \mathrm{e}-02$ & $2.3776 \mathrm{e}-02$ & $2.6554 \mathrm{e}-02$ & $2.6843 \mathrm{e}-02$ & $3.3678 \mathrm{e}-02$ & $3.3951 \mathrm{e}-02$ \\
30 & $2.7770 \mathrm{e}-02$ & $2.6094 \mathrm{e}-02$ & $2.6001 \mathrm{e}-02$ & $2.9126 \mathrm{e}-02$ & $2.9474 \mathrm{e}-02$ & $3.7124 \mathrm{e}-02$ & $3.7290 \mathrm{e}-02$ \\
40 & $2.9017 \mathrm{e}-02$ & $2.7262 \mathrm{e}-02$ & $2.7181 \mathrm{e}-02$ & $3.0495 \mathrm{e}-02$ & $3.0864 \mathrm{e}-02$ & $3.8956 \mathrm{e}-02$ & $3.9126 \mathrm{e}-02$ \\
50 & $2.9795 \mathrm{e}-02$ & $2.7989 \mathrm{e}-02$ & $2.7913 \mathrm{e}-02$ & $3.1362 \mathrm{e}-02$ & $3.1728 \mathrm{e}-02$ & $4.0095 \mathrm{e}-02$ & $4.0269 \mathrm{e}-02$ \\
60 & $3.0326 \mathrm{e}-02$ & $2.8489 \mathrm{e}-02$ & $2.8415 \mathrm{e}-02$ & $3.1954 \mathrm{e}-02$ & $3.2320 \mathrm{e}-02$ & $4.0875 \mathrm{e}-02$ & $4.1051 \mathrm{e}-02$ \\
70 & $3.0714 \mathrm{e}-02$ & $2.8853 \mathrm{e}-02$ & $2.8780 \mathrm{e}-02$ & $3.2384 \mathrm{e}-02$ & $3.2751 \mathrm{e}-02$ & $4.1444 \mathrm{e}-02$ & $4.1620 \mathrm{e}-02$ \\
80 & $3.1008 \mathrm{e}-02$ & $2.9130 \mathrm{e}-02$ & $2.9058 \mathrm{e}-02$ & $3.2711 \mathrm{e}-02$ & $3.3079 \mathrm{e}-02$ & $4.1875 \mathrm{e}-02$ & $4.2052 \mathrm{e}-02$ \\
90 & $3.1240 \mathrm{e}-02$ & $2.9348 \mathrm{e}-02$ & $2.9276 \mathrm{e}-02$ & $3.2970 \mathrm{e}-02$ & $3.3337 \mathrm{e}-02$ & $4.2213 \mathrm{e}-02$ & $4.2390 \mathrm{e}-02$ \\
100 & $3.1426 \mathrm{e}-02$ & $2.9523 \mathrm{e}-02$ & $2.9453 \mathrm{e}-02$ & $3.3178 \mathrm{e}-02$ & $3.3545 \mathrm{e}-02$ & $4.2485 \mathrm{e}-02$ & $4.2662 \mathrm{e}-02$ \\
\hline
\end{tabular}

TABLE 4.5

Total number of points close to $16000, \gamma_{j}=1 / j^{2}$.

\begin{tabular}{|r|c|cc|cc|cc|}
\hline & & $\begin{array}{c}8009 \times 2^{1} \\
=16018\end{array}$ & $\begin{array}{c}7993 \times 2^{1} \\
=15986\end{array}$ & $\begin{array}{c}4003 \times 2^{2} \\
=16012\end{array}$ & $\begin{array}{c}4001 \times 2^{2} \\
=16004\end{array}$ & $\begin{array}{c}2003 \times 2^{3} \\
=16024\end{array}$ & $\begin{array}{c}1999 \times 2^{3} \\
=15992\end{array}$ \\
\hline 10 & $7.0679 \mathrm{e}-03$ & $6.6226 \mathrm{e}-03$ & $6.7551 \mathrm{e}-03$ & $7.4726 \mathrm{e}-03$ & $7.4423 \mathrm{e}-03$ & $9.2985 \mathrm{e}-03$ & $9.3671 \mathrm{e}-03$ \\
20 & $9.7139 \mathrm{e}-03$ & $9.1387 \mathrm{e}-03$ & $9.2672 \mathrm{e}-03$ & $1.0362 \mathrm{e}-02$ & $1.0344 \mathrm{e}-02$ & $1.2975 \mathrm{e}-02$ & $1.3059 \mathrm{e}-02$ \\
30 & $1.0786 \mathrm{e}-02$ & $1.0146 \mathrm{e}-02$ & $1.0266 \mathrm{e}-02$ & $1.1513 \mathrm{e}-02$ & $1.1516 \mathrm{e}-02$ & $1.4491 \mathrm{e}-02$ & $1.4543 \mathrm{e}-02$ \\
40 & $1.1364 \mathrm{e}-02$ & $1.0691 \mathrm{e}-02$ & $1.0808 \mathrm{e}-02$ & $1.2139 \mathrm{e}-02$ & $1.2150 \mathrm{e}-02$ & $1.5307 \mathrm{e}-02$ & $1.5349 \mathrm{e}-02$ \\
50 & $1.1727 \mathrm{e}-02$ & $1.1033 \mathrm{e}-02$ & $1.1145 \mathrm{e}-02$ & $1.2528 \mathrm{e}-02$ & $1.2543 \mathrm{e}-02$ & $1.5818 \mathrm{e}-02$ & $1.5854 \mathrm{e}-02$ \\
60 & $1.1977 \mathrm{e}-02$ & $1.1268 \mathrm{e}-02$ & $1.1378 \mathrm{e}-02$ & $1.2796 \mathrm{e}-02$ & $1.2814 \mathrm{e}-02$ & $1.6168 \mathrm{e}-02$ & $1.6204 \mathrm{e}-02$ \\
70 & $1.2159 \mathrm{e}-02$ & $1.1438 \mathrm{e}-02$ & $1.1547 \mathrm{e}-02$ & $1.2993 \mathrm{e}-02$ & $1.3012 \mathrm{e}-02$ & $1.6425 \mathrm{e}-02$ & $1.6461 \mathrm{e}-02$ \\
80 & $1.2299 \mathrm{e}-02$ & $1.1567 \mathrm{e}-02$ & $1.1676 \mathrm{e}-02$ & $1.3143 \mathrm{e}-02$ & $1.3163 \mathrm{e}-02$ & $1.6622 \mathrm{e}-02$ & $1.6657 \mathrm{e}-02$ \\
90 & $1.2409 \mathrm{e}-02$ & $1.1670 \mathrm{e}-02$ & $1.1778 \mathrm{e}-02$ & $1.3261 \mathrm{e}-02$ & $1.3282 \mathrm{e}-02$ & $1.6778 \mathrm{e}-02$ & $1.6812 \mathrm{e}-02$ \\
100 & $1.2498 \mathrm{e}-02$ & $1.1753 \mathrm{e}-02$ & $1.1861 \mathrm{e}-02$ & $1.3357 \mathrm{e}-02$ & $1.3379 \mathrm{e}-02$ & $1.6904 \mathrm{e}-02$ & $1.6938 \mathrm{e}-02$ \\
\hline
\end{tabular}

TABLE 4.6

Total number of points close to $64000, \gamma_{j}=1 / j^{2}$.

\begin{tabular}{|r|c|cc|cc|cc|}
\hline$d$ & 64007 & $\begin{array}{c}32009 \times 2^{1} \\
=64018\end{array}$ & $\begin{array}{c}32003 \times 2^{1} \\
=64006\end{array}$ & $\begin{array}{c}16007 \times 2^{2} \\
=64028\end{array}$ & $\begin{array}{c}16001 \times 2^{2} \\
=64004\end{array}$ & $\begin{array}{c}8009 \times 2^{3} \\
=64072\end{array}$ & $\begin{array}{c}7993 \times 2^{3} \\
=63944\end{array}$ \\
\hline 10 & $2.5983 \mathrm{e}-03$ & $2.4131 \mathrm{e}-03$ & $2.4454 \mathrm{e}-03$ & $2.6945 \mathrm{e}-03$ & $2.6743 \mathrm{e}-03$ & $3.3548 \mathrm{e}-03$ & $3.3135 \mathrm{e}-03$ \\
20 & $3.7412 \mathrm{e}-03$ & $3.5099 \mathrm{e}-03$ & $3.5290 \mathrm{e}-03$ & $3.9372 \mathrm{e}-03$ & $3.9465 \mathrm{e}-03$ & $4.9385 \mathrm{e}-03$ & $4.9097 \mathrm{e}-03$ \\
30 & $4.2141 \mathrm{e}-03$ & $3.9582 \mathrm{e}-03$ & $3.9767 \mathrm{e}-03$ & $4.4501 \mathrm{e}-03$ & $4.4667 \mathrm{e}-03$ & $5.5924 \mathrm{e}-03$ & $5.5795 \mathrm{e}-03$ \\
40 & $4.4705 \mathrm{e}-03$ & $4.2019 \mathrm{e}-03$ & $4.2200 \mathrm{e}-03$ & $4.7333 \mathrm{e}-03$ & $4.7496 \mathrm{e}-03$ & $5.9532 \mathrm{e}-03$ & $5.9446 \mathrm{e}-03$ \\
50 & $4.6325 \mathrm{e}-03$ & $4.3564 \mathrm{e}-03$ & $4.3735 \mathrm{e}-03$ & $4.9111 \mathrm{e}-03$ & $4.9270 \mathrm{e}-03$ & $6.1803 \mathrm{e}-03$ & $6.1750 \mathrm{e}-03$ \\
60 & $4.7448 \mathrm{e}-03$ & $4.4624 \mathrm{e}-03$ & $4.4798 \mathrm{e}-03$ & $5.0334 \mathrm{e}-03$ & $5.0484 \mathrm{e}-03$ & $6.3377 \mathrm{e}-03$ & $6.3334 \mathrm{e}-03$ \\
70 & $4.8270 \mathrm{e}-03$ & $4.5399 \mathrm{e}-03$ & $4.5573 \mathrm{e}-03$ & $5.1227 \mathrm{e}-03$ & $5.1371 \mathrm{e}-03$ & $6.4533 \mathrm{e}-03$ & $6.4493 \mathrm{e}-03$ \\
80 & $4.8901 \mathrm{e}-03$ & $4.5991 \mathrm{e}-03$ & $4.6166 \mathrm{e}-03$ & $5.1912 \mathrm{e}-03$ & $5.2052 \mathrm{e}-03$ & $6.5416 \mathrm{e}-03$ & $6.5378 \mathrm{e}-03$ \\
90 & $4.9398 \mathrm{e}-03$ & $4.6460 \mathrm{e}-03$ & $4.6635 \mathrm{e}-03$ & $5.2452 \mathrm{e}-03$ & $5.2593 \mathrm{e}-03$ & $6.6113 \mathrm{e}-03$ & $6.6077 \mathrm{e}-03$ \\
100 & $4.9801 \mathrm{e}-03$ & $4.6840 \mathrm{e}-03$ & $4.7014 \mathrm{e}-03$ & $5.2890 \mathrm{e}-03$ & $5.3032 \mathrm{e}-03$ & $6.6678 \mathrm{e}-03$ & $6.6645 \mathrm{e}-03$ \\
\hline
\end{tabular}

decays more slowly than $1,1 / 4,1 / 9, \ldots$, and so in the former case, the third variable is still fairly important, while this is not the situation in the latter case.

The phenomenon is also supported by our earlier analysis. Since it may be verified numerically that (2.7) holds, Theorem 2.3 and Lemma 2.4 together suggest that it would be advantageous to copy in the first $r$ dimensions if $\ell=2, \alpha=2$, and

$$
\frac{\gamma_{r}}{\beta_{r}}>\frac{6}{\pi^{2}} \approx 0.6079
$$

For $\gamma_{j}=0.9^{j}$, this is obviously satisfied when $r=1, r=2$, and $r=3$. For $\gamma_{j}=1 / j^{2}$, this is satisfied only when $r=1$. Because Lemma 2.4 provides only a sufficient 
TABLE 4.7

Ratios of worst-case errors at $d=100$.

\begin{tabular}{|c|c|cc|cc|cc|}
\hline & Approximate $N$ & \multicolumn{2}{|c|}{$r=1$} & \multicolumn{2}{|c|}{$r=2$} & \multicolumn{2}{c|}{$r=3$} \\
\hline \multirow{3}{*}{$\gamma_{j}=0.9^{j}$} & 4000 & 0.941 & 0.941 & 0.895 & 0.901 & 0.871 & 0.874 \\
& 16000 & 0.944 & 0.946 & 0.903 & 0.904 & 0.877 & 0.878 \\
& 64000 & 0.947 & 0.945 & 0.904 & 0.904 & 0.878 & 0.879 \\
\hline \multirow{3}{*}{$\gamma_{j}=1 / j^{2}$} & 4000 & 0.939 & 0.937 & 1.056 & 1.067 & 1.352 & 1.358 \\
& 16000 & 0.940 & 0.949 & 1.069 & 1.070 & 1.353 & 1.355 \\
& 64000 & 0.941 & 0.944 & 1.062 & 1.065 & 1.339 & 1.338 \\
\hline
\end{tabular}

TABLE 4.8

Values of $\rho_{2, r}$ for $r=1,2,3$.

\begin{tabular}{|c|cc|cc|cc|}
\hline & $\rho_{2,1}$ & $\sqrt{\rho_{2,1}}$ & $\rho_{2,2}$ & $\sqrt{\rho_{2,2}}$ & $\rho_{2,3}$ & $\sqrt{\rho_{2,3}}$ \\
\hline$\gamma_{j}=0.9^{j}$ & 0.879 & 0.937 & 0.799 & 0.894 & 0.752 & 0.867 \\
$\gamma_{j}=1 / j^{2}$ & 0.850 & 0.922 & 1.124 & 1.060 & 1.797 & 1.340 \\
\hline
\end{tabular}

condition for $\rho_{2, r}$ to be less than one, a direct calculation of $\rho_{2, r}$ was done, and the results (see Table 4.8) show the same conclusion.

If we compare the values of $\sqrt{\rho_{2, r}}$ in Table 4.8 with the ratios in Table 4.7, we see that the values of $\sqrt{\rho_{2, r}}$ are reasonably close to the ratios. So, although $\rho_{2, r}$ is essentially a ratio of means, there is numerical evidence here that it provides a measure of the ratios of the square worst-case errors obtained from intermediate-rank lattice rules and rank-1 lattice rules in the weighted Korobov space setting.

For our choices of weights, it follows from Theorem 2.7 that the rate of convergence is $O\left(N^{-1+\delta}\right)$ for $\delta>0$, independently of the dimension $d$. However, the numerical results presented show a rate of convergence of roughly $O\left(N^{-1 / 2}\right)$ for the case $\gamma_{j}=$ $0.9^{j}$ and a somewhat better rate for the case $\gamma_{j}=1 / j^{2}$. The observed rates of convergence also appear to be higher for the smaller values of $d$. This agrees with the numerical results in [9], where the predicted rate of convergence is not observed when moderate values of $n$ are used relative to the dimension. In that situation, the observed rate of convergence depends on the rate of decay of the weights, with faster decaying weights yielding higher convergence rates. To get an observed rate of convergence close to $O\left(N^{-1}\right)$, we need to have weights that decay much faster, for example, $\gamma_{j}=0.1^{j}$ or $\gamma_{j}=1 / j^{6}$. However, if weights such as these were used, the theory would suggest that there would not be much benefit in doing any copying.

Appendix. Let $\left(1, z_{2}, \ldots, z_{d}\right)$ be constructed using Algorithm 2.5. Here we prove Theorem 2.6; that is, for each $s=1,2, \ldots, d$, we have

$$
e_{n, s, \operatorname{copy}(\ell, \min (s, r))}^{2}\left(1, z_{2}, \ldots, z_{s}\right) \leq(n-1)^{-\frac{1}{\lambda}} \prod_{j=1}^{s}\left(\beta_{j}^{\lambda}+2 \bar{\gamma}_{j}^{\lambda} \zeta(\alpha \lambda)\right)^{\frac{1}{\lambda}}
$$

for all $\lambda$ satisfying $\frac{1}{\alpha}<\lambda \leq 1$, where

$$
\bar{\gamma}_{j}:= \begin{cases}\frac{\gamma_{j}}{\ell^{\alpha}} & \text { if } 1 \leq j \leq r \\ \gamma_{j} & \text { otherwise. }\end{cases}
$$

The proof makes use of one form of Jensen's inequality (see Theorem 19 of [2]), which states that for $\left\{a_{i}\right\}$ a sequence of positive numbers,

$$
\sum a_{i} \leq\left(\sum a_{i}^{\lambda}\right)^{\frac{1}{\lambda}} \quad \text { for } 0<\lambda \leq 1
$$


Proof. For $s=1$, it is not hard to show that for all $z_{1}$ we have

$$
e_{n, 1, \operatorname{copy}(\ell, 1)}^{2}\left(z_{1}\right)=\frac{2 \bar{\gamma}_{1} \zeta(\alpha)}{n^{\alpha}},
$$

and for any $\lambda$ satisfying $\frac{1}{\alpha}<\lambda \leq 1$, we have

$$
\frac{2 \bar{\gamma}_{1} \zeta(\alpha)}{n^{\alpha}} \leq n^{-\alpha}\left(\beta_{1}+2 \bar{\gamma}_{1} \zeta(\alpha)\right) \leq n^{-\alpha}\left(\beta_{1}^{\lambda}+2^{\lambda} \bar{\gamma}_{1}^{\lambda}[\zeta(\alpha)]^{\lambda}\right)^{\frac{1}{\lambda}},
$$

where the second inequality follows by applying Jensen's inequality to the sum $\beta_{1}+$ $2 \bar{\gamma}_{1} \zeta(\alpha)$. It can be easily verified that $n^{-\alpha}<(n-1)^{-\frac{1}{\lambda}}, 2^{\lambda}<2$, and by Jensen's inequality, $[\zeta(\alpha)]^{\lambda} \leq \zeta(\alpha \lambda)$. Hence the result holds for $s=1$.

For $s$ satisfying $2 \leq s \leq d$, suppose that an $(s-1)$-dimensional vector $\left(1, z_{2}, \ldots, z_{s-1}\right)$ has already been constructed using Algorithm 2.5 such that it satisfies

$$
e_{n, s-1, \operatorname{copy}(\ell, \min (s-1, r))}^{2}\left(1, z_{2}, \ldots, z_{s-1}\right) \leq(n-1)^{-\frac{1}{\lambda}} \prod_{j=1}^{s-1}\left(\beta_{j}^{\lambda}+2 \bar{\gamma}_{j}^{\lambda} \zeta(\alpha \lambda)\right)^{\frac{1}{\lambda}}
$$

for all $\lambda$ satisfying $\frac{1}{\alpha}<\lambda \leq 1$. For any $z_{s} \in \mathbb{Z}_{n}$, there is a corresponding $\bar{z}_{s}$ (recall that $\bar{z}_{s}=\ell z_{s}$ if $s \leq r$ and $\bar{z}_{s}=z_{s}$ if $s>r$ ), and it follows from (2.6) that

$$
\begin{aligned}
& e_{n, s, \operatorname{copy}(\ell, \min (s, r))}^{2}\left(1, z_{2}, \ldots, z_{s}\right) \\
& (\text { A.2 })=\beta_{s} e_{n, s-1, \operatorname{copy}(\ell, \min (s-1, r))}^{2}\left(1, z_{2}, \ldots, z_{s-1}\right)+\theta_{n, s}\left(\alpha, \boldsymbol{\beta}, \bar{\gamma} ; 1, z_{2}, \ldots, z_{s}\right),
\end{aligned}
$$

where

$$
\begin{aligned}
& \theta_{n, s}\left(\alpha, \boldsymbol{\beta}, \overline{\boldsymbol{\gamma}} ; 1, z_{2}, \ldots, z_{s}\right) \\
& =\frac{\bar{\gamma}_{s}}{n} \sum_{k=0}^{n-1}\left[\prod_{j=1}^{s-1}\left(\beta_{j}+\bar{\gamma}_{j} \sum_{h=-\infty}^{\infty} \frac{e^{2 \pi \mathrm{i} h k \bar{z}_{j} / n}}{|h|^{\alpha}}\right) \sum_{h=-\infty}^{\infty} \frac{e^{2 \pi \mathrm{i} h k \bar{z}_{s} / n}}{|h|^{\alpha}}\right] .
\end{aligned}
$$

Later we shall prove the following:

(i) For given $\alpha, \boldsymbol{\beta}$, and $\overline{\boldsymbol{\gamma}}$, there exists $z_{s}=z_{s}(\alpha, \boldsymbol{\beta}, \bar{\gamma})$ such that

$$
\theta_{n, s}\left(\alpha, \boldsymbol{\beta}, \bar{\gamma} ; 1, z_{2}, \ldots, z_{s}\right) \leq \frac{2 \bar{\gamma}_{s} \zeta(\alpha)}{n-1} \prod_{j=1}^{s-1}\left(\beta_{j}+2 \bar{\gamma}_{j} \zeta(\alpha)\right)
$$

(ii) For all $\frac{1}{\alpha}<\lambda \leq 1$,

$$
\theta_{n, s}\left(\alpha, \boldsymbol{\beta}, \overline{\boldsymbol{\gamma}} ; 1, z_{2}, \ldots, z_{s}\right) \leq\left[\theta_{n, s}\left(\alpha \lambda, \boldsymbol{\beta}^{\lambda}, \overline{\boldsymbol{\gamma}}^{\lambda} ; 1, z_{2}, \ldots, z_{s}\right)\right]^{\frac{1}{\lambda}}
$$

where $\boldsymbol{\beta}^{\lambda}=\left\{\beta_{j}^{\lambda}\right\}$ and $\overline{\boldsymbol{\gamma}}^{\lambda}=\left\{\bar{\gamma}_{j}^{\lambda}\right\}$.

We see from (i) with $\alpha, \boldsymbol{\beta}$, and $\overline{\boldsymbol{\gamma}}$ replaced by $\alpha \lambda, \boldsymbol{\beta}^{\lambda}$, and $\overline{\boldsymbol{\gamma}}^{\lambda}$, respectively, that there exists $z_{s}=z_{s}\left(\alpha \lambda, \boldsymbol{\beta}^{\lambda}, \bar{\gamma}^{\lambda}\right)$ such that

$$
\theta_{n, s}\left(\alpha \lambda, \boldsymbol{\beta}^{\lambda}, \bar{\gamma}^{\lambda} ; 1, z_{2}, \ldots, z_{s}\right) \leq \frac{2 \bar{\gamma}_{s}^{\lambda} \zeta(\alpha \lambda)}{n-1} \prod_{j=1}^{s-1}\left(\beta_{j}^{\lambda}+2 \bar{\gamma}_{j}^{\lambda} \zeta(\alpha \lambda)\right)
$$


For this $z_{s}=z_{s}\left(\alpha \lambda, \boldsymbol{\beta}^{\lambda}, \overline{\boldsymbol{\gamma}}^{\lambda}\right)$, it then follows from (ii) that

$$
\theta_{n, s}\left(\alpha, \boldsymbol{\beta}, \overline{\boldsymbol{\gamma}} ; 1, z_{2}, \ldots, z_{s}\right) \leq \frac{2^{\frac{1}{\lambda}} \bar{\gamma}_{s}[\zeta(\alpha \lambda)]^{\frac{1}{\lambda}}}{(n-1)^{\frac{1}{\lambda}}} \prod_{j=1}^{s-1}\left(\beta_{j}^{\lambda}+2 \bar{\gamma}_{j}^{\lambda} \zeta(\alpha \lambda)\right)^{\frac{1}{\lambda}} .
$$

Thus it follows from (A.1) and (A.2) that this $z_{s}=z_{s}\left(\alpha \lambda, \boldsymbol{\beta}^{\lambda}, \bar{\gamma}^{\lambda}\right)$ satisfies

$$
\begin{aligned}
& e_{n, s, \operatorname{copy}(\ell, \min (s, r))}^{2}\left(1, z_{2}, \ldots, z_{s}\right) \\
& \leq\left(\beta_{s}+2^{\frac{1}{\lambda}} \bar{\gamma}_{s}[\zeta(\alpha \lambda)]^{\frac{1}{\lambda}}\right)(n-1)^{-\frac{1}{\lambda}} \prod_{j=1}^{s-1}\left(\beta_{j}^{\lambda}+2 \bar{\gamma}_{j}^{\lambda} \zeta(\alpha \lambda)\right)^{\frac{1}{\lambda}} \\
& \leq\left(\beta_{s}^{\lambda}+2 \bar{\gamma}_{s}^{\lambda} \zeta(\alpha \lambda)\right)^{\frac{1}{\lambda}}(n-1)^{-\frac{1}{\lambda}} \prod_{j=1}^{s-1}\left(\beta_{j}^{\lambda}+2 \bar{\gamma}_{j}^{\lambda} \zeta(\alpha \lambda)\right)^{\frac{1}{\lambda}} \\
& =(n-1)^{-\frac{1}{\lambda}} \prod_{j=1}^{s}\left(\beta_{j}^{\lambda}+2 \bar{\gamma}_{j}^{\lambda} \zeta(\alpha \lambda)\right)^{\frac{1}{\lambda}},
\end{aligned}
$$

where the second inequality follows from applying Jensen's inequality to the sum in the first factor. Now since we choose $z_{s}$ in Algorithm 2.5 to minimize the square worst-case error $e_{n, s, \operatorname{copy}(\ell, \min (s, r))}^{2}\left(1, z_{2}, \ldots, z_{s}\right)$, this choice of $z_{s}$ must satisfy the same bound. Hence it follows inductively that the result holds for all $s=2,3, \ldots, d$.

To complete the proof, we need to prove (i) and (ii).

Proof of (i). Clearly there exists a $z_{s}=z_{s}(\alpha, \boldsymbol{\beta}, \bar{\gamma})$ (and hence $\bar{z}_{s}$ ) such that

$$
\begin{aligned}
& \theta_{n, s}\left(\alpha, \boldsymbol{\beta}, \bar{\gamma} ; 1, z_{2}, \ldots, z_{s}\right) \\
\leq & \frac{1}{n-1} \sum_{z_{s}=1}^{n-1} \theta_{n, s}\left(\alpha, \boldsymbol{\beta}, \bar{\gamma} ; 1, z_{2}, \ldots, z_{s}\right) \\
(\mathrm{A} .4)= & \frac{\bar{\gamma}_{s}}{n} \sum_{k=0}^{n-1}\left[\prod_{j=1}^{s-1}\left(\beta_{j}+\bar{\gamma}_{j} \sum_{h=-\infty}^{\infty} \frac{e^{2 \pi \mathrm{i} h k \bar{z}_{j} / n}}{|h|^{\alpha}}\right)\left(\frac{1}{n-1} \sum_{z_{s}=1}^{n-1} \sum_{h=-\infty}^{\infty} \frac{e^{2 \pi \mathrm{i} h k \bar{z}_{s} / n}}{|h|^{\alpha}}\right)\right] .
\end{aligned}
$$

Since $n$ is prime and $\operatorname{gcd}(\ell, n)=1$, it can be shown for $q=1$ and $q=\ell$ that

$$
\frac{1}{n-1} \sum_{z=1}^{n-1} \sum_{h=-\infty}^{\infty} \frac{e^{2 \pi \mathrm{i} h k q z / n}}{|h|^{\alpha}}= \begin{cases}2 \zeta(\alpha) & \text { if } k \text { is a multiple of } n \\ -\frac{2 \zeta(\alpha)\left(1-n^{1-\alpha}\right)}{n-1} & \text { otherwise. }\end{cases}
$$

Upon separating out the $k=0$ term and using the result above, (A.4) becomes

$$
\frac{2 \bar{\gamma}_{s} \zeta(\alpha)}{n} \prod_{j=1}^{s-1}\left(\beta_{j}+2 \bar{\gamma}_{j} \zeta(\alpha)\right)-\frac{2 \bar{\gamma}_{s} \zeta(\alpha)\left(1-n^{1-\alpha}\right)}{n(n-1)} \sum_{k=1}^{n-1} \prod_{j=1}^{s-1}\left(\beta_{j}+\bar{\gamma}_{j} \sum_{h=-\infty}^{\infty} \frac{e^{2 \pi \mathrm{i} h k \bar{z}_{j} / n}}{|h|^{\alpha}}\right) .
$$

It follows from (2.6) (with the $k=0$ term separated out) that

$$
\begin{aligned}
& \frac{1}{n} \sum_{k=1}^{n-1} \prod_{j=1}^{s-1}\left(\beta_{j}+\bar{\gamma}_{j} \sum_{h=-\infty}^{\infty} \frac{e^{2 \pi \mathrm{i} h k \bar{z}_{j} / n}}{|h|^{\alpha}}\right) \\
& =e_{n, s-1, \operatorname{copy}(\ell, \min (s-1, r))}^{2}\left(1, z_{2}, \ldots, z_{s-1}\right)+\prod_{j=1}^{s-1} \beta_{j}-\frac{1}{n} \prod_{j=1}^{s-1}\left(\beta_{j}+2 \bar{\gamma}_{j} \zeta(\alpha)\right) .
\end{aligned}
$$


Hence there exists a $z_{s}=z_{s}(\alpha, \boldsymbol{\beta}, \bar{\gamma})$ such that

$$
\begin{aligned}
& \theta_{n, s}\left(\alpha, \boldsymbol{\beta}, \bar{\gamma} ; 1, z_{2}, \ldots, z_{s}\right) \\
& \leq \frac{2 \bar{\gamma}_{s} \zeta(\alpha)}{n} \prod_{j=1}^{s-1}\left(\beta_{j}+2 \bar{\gamma}_{j} \zeta(\alpha)\right)+\frac{2 \bar{\gamma}_{s} \zeta(\alpha)\left(1-n^{1-\alpha}\right)}{n(n-1)} \prod_{j=1}^{s-1}\left(\beta_{j}+2 \bar{\gamma}_{j} \zeta(\alpha)\right) \\
& \leq \frac{2 \bar{\gamma}_{s} \zeta(\alpha)}{n}\left(1+\frac{1}{n-1}\right) \prod_{j=1}^{s-1}\left(\beta_{j}+2 \bar{\gamma}_{j} \zeta(\alpha)\right) \\
& =\frac{2 \bar{\gamma}_{s} \zeta(\alpha)}{(n-1)} \prod_{j=1}^{s-1}\left(\beta_{j}+2 \bar{\gamma}_{j} \zeta(\alpha)\right) .
\end{aligned}
$$

Proof of (ii). Let

$$
r(\alpha, \beta, \gamma, h):= \begin{cases}\beta^{-1} & \text { if } h=0 \\ \gamma^{-1}|h|^{\alpha} & \text { if } h \neq 0 .\end{cases}
$$

With this notation we can write $\theta_{n, s}\left(\alpha, \boldsymbol{\beta}, \overline{\boldsymbol{\gamma}} ; 1, z_{2}, \ldots, z_{s}\right)$ in (A.3) as

$$
\begin{aligned}
& \theta_{n, s}\left(\alpha, \boldsymbol{\beta}, \overline{\boldsymbol{\gamma}} ; 1, z_{2}, \ldots, z_{s}\right) \\
& =\frac{\bar{\gamma}_{s}}{n} \sum_{\substack{k=0 \\
n-1}} \sum_{\substack{\boldsymbol{h} \in \mathbb{Z}^{s} \\
h_{s} \neq 0}} \frac{e^{2 \pi \mathrm{i} k\left(h_{1}, h_{2}, \ldots, h_{s}\right) \cdot\left(\bar{z}_{1}, \bar{z}_{2}, \ldots, \bar{z}_{s}\right) / n}}{\left|h_{s}\right|^{\alpha} \prod_{j=1}^{s-1} r\left(\alpha, \beta_{j}, \bar{\gamma}_{j}, h_{j}\right)} \\
& =\bar{\gamma}_{s} \sum_{\substack{\boldsymbol{h} \in \mathbb{Z}^{s} \\
h_{s} \neq 0 \\
\left(h_{1}, h_{2}, \ldots, h_{s}\right) \cdot\left(\bar{z}_{1}, \bar{z}_{2}, \ldots, \bar{z}_{s}\right) \equiv 0(\bmod n)}}\left(\left|h_{s}\right|^{-\alpha} \prod_{j=1}^{s-1} r\left(\alpha, \beta_{j}, \bar{\gamma}_{j}, h_{j}\right)^{-1}\right)
\end{aligned}
$$

since

$$
\sum_{k=0}^{n-1} e^{2 \pi \mathrm{i} k\left(h_{1}, h_{2}, \ldots, h_{s}\right) \cdot\left(\bar{z}_{1}, \bar{z}_{2}, \ldots, \bar{z}_{s}\right) / n}=\sum_{k=0}^{n-1}\left(e^{2 \pi \mathrm{i}\left(h_{1}, h_{2}, \ldots, h_{s}\right) \cdot\left(\bar{z}_{1}, \bar{z}_{2}, \ldots, \bar{z}_{s}\right) / n}\right)^{k}=0
$$

if $\left(h_{1}, h_{2}, \ldots, h_{s}\right) \cdot\left(\bar{z}_{1}, \bar{z}_{2}, \ldots, \bar{z}_{s}\right)$ is not a multiple of $n$. It now follows from Jensen's inequality that

$$
\begin{aligned}
& \theta_{n, s}\left(\alpha, \boldsymbol{\beta}, \overline{\boldsymbol{\gamma}} ; 1, z_{2}, \ldots, z_{s}\right) \\
& \leq \bar{\gamma}_{s}\left[\sum_{\substack{\boldsymbol{h} \in \mathbb{Z}^{s} \\
h_{s} \neq 0 \\
\left(h_{1}, h_{2}, \ldots, h_{s}\right) \cdot\left(\bar{z}_{1}, \bar{z}_{2}, \ldots, \bar{z}_{s}\right) \equiv 0(\bmod n)}}\left(\left|h_{s}\right|^{-\alpha \lambda} \prod_{j=1}^{s-1} r\left(\alpha, \beta_{j}, \bar{\gamma}_{j}, h_{j}\right)^{-\lambda}\right)\right]^{\frac{1}{\lambda}} \\
& =\left[\theta_{n, s}\left(\alpha \lambda, \boldsymbol{\beta}^{\lambda}, \overline{\boldsymbol{\gamma}}^{\lambda} ; 1, z_{2}, \ldots, z_{s}\right)\right]^{\frac{1}{\lambda}},
\end{aligned}
$$

where the last step follows from the property $r(\alpha, \beta, \gamma, h)^{\lambda}=r\left(\alpha \lambda, \beta^{\lambda}, \gamma^{\lambda}, h\right)$. This completes the proof. 


\section{REFERENCES}

[1] J. Dick, On the convergence rate of the component-by-component construction of good lattice rules, J. Complexity, submitted.

[2] G. H. Hardy, J. E. Littlewood, and G. Pólya, Inequalities, Cambridge University Press, Cambridge, UK, 1934.

[3] F. J. Hickernell ANd H. S. Hong, Quasi-Monte Carlo methods and their randomizations, in Applied Probability, AMS/IP Stud. Adv. Math. 26, R. Chan, Y.-K. Kwok, D. Yao, and Q Zhang, eds., AMS, Providence, RI, 2002, pp. 59-77.

[4] F. J. Hickernell And H. Niederreiter, The existence of good extensible rank-1 lattices, J. Complexity, 19 (2003), pp. 286-300.

5] F. J. Hickernell AND H. WoźNiAKOWSKI, Integration and approximation in arbitrary dimensions, Adv. Comput. Math., 12 (2000), pp. 25-58.

[6] F. J. Hickernell AND H. WoźNiakowski, Tractability of multivariate integration for periodic functions, J. Complexity, 17 (2001), pp. 660-682.

[7] S. Joe And S. A. R. Disney, Intermediate rank lattice rules for multidimensional integration, SIAM J. Numer. Anal., 30 (1993), pp. 569-582.

[8] S. Joe AND I. H. SloAn, Imbedded lattice rules for multidimensional integration, SIAM J. Numer. Anal., 29 (1992), pp. 1119-1135.

[9] F. Y. KuO, Component-by-component constructions achieve the optimal rate of convergence for multivariate integration in weighted Korobov and Sobolev spaces, J. Complexity, 19 (2003), pp. 301-320.

[10] F. Y. KUO AND S. JoE, Component-by-component construction of good QMC rules with a composite number of quadrature points, J. Complexity, 18 (2002), pp. 943-976.

[11] I. H. SloAn And S. Joe, Lattice Rules for Multiple Integration, Clarendon Press, Oxford, UK, 1994.

[12] I. H. Sloan, F. Y. Kuo, And S. Joe, On the step-by-step construction of quasi-Monte Carlo integration rules that achieve strong tractability error bounds in weighted Sobolev spaces, Math. Comp., 71 (2002), pp. 1609-1640.

[13] I. H. Sloan, F. Y. Kuo, And S. Joe, Constructing randomly shifted lattice rules in weighted Sobolev spaces, SIAM J. Numer. Anal., 40 (2002), pp. 1650-1665.

[14] I. H. SloAn AND J. N. Lyness, The representation of lattice quadrature rules as multiple sums, Math. Comp., 52 (1989), pp. 81-94.

[15] I. H. SloAn AND A. V. RezTsov, Component-by-component construction of good lattice rules, Math. Comp., 71 (2002), pp. 263-273.

[16] I. H. Sloan and H. WoźNIAKOWSKi, Tractability of multivariate integration for weighted Korobov classes, J. Complexity, 17 (2001), pp. 697-721. 